\title{
EL DESCENSO DE LA MORTALIDAD INFANTIL EN MADRID, 1900-1970*
}

Rosa Gómez Redondo

\section{INTRODUCCION}

El estudio del descenso de la mortalidad infantil apenas precisa justificación. Baste decir que si el descenso sostenido de la mortalidad es un fenómeno de capital importancia en la historia de la humanidad por ser el origen del crecimiento moderno de la población ${ }^{1}$, cuando se trata de la caída de la mortalidad infantil adquiere especial interés por la alta proporción que representa esta mortalidad específica dentro de la general. Asimismo, el aumento de la esperanza de vida al nacer producido por este descenso tiene consecuencias sobre el volumen y estructura de la población que revisten singular trascendencia.

Por otra parte, el estudio de diferentes países ha puesto de manifiesto que el descenso de la mortalidad infantil se debe a cambios en el entorno en que se produce ésta y, por ello, el nivel y características de la mortalidad infantil,

* El tema de este artículo fue desarrollado en la Memoria de Licenciatura de la autora -El descenso de la mortalidad infantil en Madrid, 1900-1970-, presentada en la Facultad de CC. PP. y Sociología en 1983, bajo la dirección del doctor Joaquín Arango VilaBelda, a quien también la autora agradece la revisión crítica del presente artículo.

${ }^{1}$ Expresión referida al aumento demográfico durante los últimos doscientos cincuenta o trescientos años, que da título a la obra de Th. MACKEOwn, El crecimiento moderno de la población, Barcelona, Antoni Bosch (editor), 1978, p. 1. 
además de medir el desarrollo demográfico de una nación, se ha convertido en un indicador social de primera importancia.

En España, tan trascendental proceso no ha sido suficientemente estudiado y, cuando se ha hecho, generalmente el análisis ha sido limitado a periodos cortos, no superando la mera descripción numérica del descenso ${ }^{2}$. Dado que con frecuencia los estudios a nivel nacional han demostrado ser escasamente representativos de las unidades geográfico-administrativas que comprende esa nación, debido a la diversidad de situaciones económicas, culturales y demográficas de que disfrutan aquéllas, y antes de abordar el estudio de este fenómeno para toda España —en su desagregación provincial-, quizá convenga realizarlo en un marco reducido, Madrid.

El estudio de esta capital se compara con el de su entorno - el resto de la provincia- La provincia elegida es un medio heterogéneo con acusadas disparidades entre la capital y el resto de la provincia, lo que hace posible la confrontación entre el medio predominantemente urbano y rural durante buena parte del período. Sin embargo, no es posible identificar estrictamente ambos medios con dichas categorías ecológicas, ya que de hacerlo se infravaloraría el medio urbano y, por tanto, se sobrevaloraría el medio rural de la provincia. De haber sido posible, la división de la provincia en medio rural y medio urbano tendría que haberse basado en los datos de mortalidad infantil desagregados por municipios (de acuerdo con las características de éstos), pero los datos necesarios para la realización del estudio presente no son facilitados por las fuentes estadísticas oficiales, a ese nivel de desagregación, para el período propuesto.

Asimismo, la división de la provincia en capital y resto es necesaria a fin de evitar el sesgo que el peso mayoritario de la población de la capital introduce sobre el total provincial.

El estudio de la mortalidad infantil en la sociedad madrileña se ve completado con el paralelo de la española en su conjunto, con el fin de contar con un marco de referencia que permita evaluar el nivel del fenómeno y el ritmo del proceso.

La elección del período, 1900-1970, responde a dos razones. La extensión es aconsejable por razones metodológicas, ya que sólo en base a períodos largos es posible discernir la verdadera tendencia de un fenómeno demográfico y sus alteraciones coyunturales. Por otra parte, si el objetivo de la investigación realizada es conocer el descenso de la mortalidad infantil, ésos son los años fundamentales a estudiar, puesto que en su transcurso se produce el grueso de los grandes cambios cuantitativos, así como importantes cambios cualitativos, en aquel fenómeno demográfico. Con anterioridad a estas fechas,

2 Sólo A. Arbelo Curbelo trata el tema monográficamente, cubriendo el período completo en el que se produce el grueso del descenso de la mortalidad infantil, pero su visión es fundamentalmente médica. La mortalidad de la infancia en España, 1901-1950, Madrid, Consejo Superior de Investigaciones Científicas, 1962, pp. 65-306, y Demografía sanitaria infantil, Madrid, Ed. Paz Montalvo, 1980, pp. 391-441 y 475-536. 
si bien el descenso en la mortalidad general ya era importante, en los menores de un año de edad es aún insignificante.

Estudiar el descenso de la mortalidad infantil supone conocer tanto su cronología como la evolución seguida por sus componentes desde diferentes perspectivas: desde el punto de vista ecológico (capital-resto de provincia), de la etiología del fallecimiento (mortalidad por causa endógena y exógena) y de la edad del fallecido (mortalidad neonatal y postneonatal), así como las relaciones existentes entre ellos.

La fuente de datos utilizada fundamentalmente en este estudio es el Movimiento Natural de Población de España, publicado por el Instituto Nacional de Estadística (INE) ${ }^{3}$; sin embargo, en dichos datos se han observado errores, que se expondrán más adelante.

Por último, la evolución del fenómeno estudiado se ha analizado a partir de tasas corregidas, para evitar el error que introduce en la medición de esta mortalidad específica el peculiar concepto de «nacido vivo» del Código Civil español.

Este artículo se ocupa solamente del estudio demográfico de la mortalidad infantil. Queda para otra ocasión abordar las complejas interrelaciones de este fenómeno con otras variables demográficas, especialmente con la fecundidad, así como con variables no demográficas (alimentación, urbanización, alfabetización y educación e infraestructura higiénico-sanitaria, fundamentalmente), para determinar las causas del descenso del fenómeno analizado.

\section{LA EVOLUCION DE LA MORTALIDAD INFANTIL}

\section{El descenso de la mortalidad infantil}

El análisis de la evolución de la mortalidad infantil en Madrid de 1900 a 1970 evidencia un extraordinario descenso de la misma a lo largo del período. De cada 100 defunciones de niños menores de un año de edad que se producían en torno a 1900 , setenta años más tarde se evitaban nada menos que 88. Algo parecido, aunque en una proporción inferior, puede decirse del resto de la provincia (descenso de un 80 por 100) y del total de España (85 por 100$)$.

La tendencia y el ritmo del descenso de la mortalidad infantil en Madrid corren paralelos a los seguidos por España en su conjunto (cuadro 1 y gráfico 1). Respecto a los niveles de esta mortalidad, en la capital están por debajo de la media nacional a lo largo del período, salvo en los primeros veinticinco

${ }^{3}$ Los Movimientos Naturales de la Población de España consultados —desde 1900 a 1970 - son publicados por diferentes organismos hasta que, a partir de 1944 y hasta la actualidad, lo son por el Instituto Nacional de Estadística. 


\section{CUADRO 1}

Tasas de mortalidad infantil corregidas $\left(T M I_{C}\right)(1)$

$y$ legales $\left(T M I_{L}\right)(2), 1900-1970$

Tasas $(1 \times 1.000$ nacidos vivos)

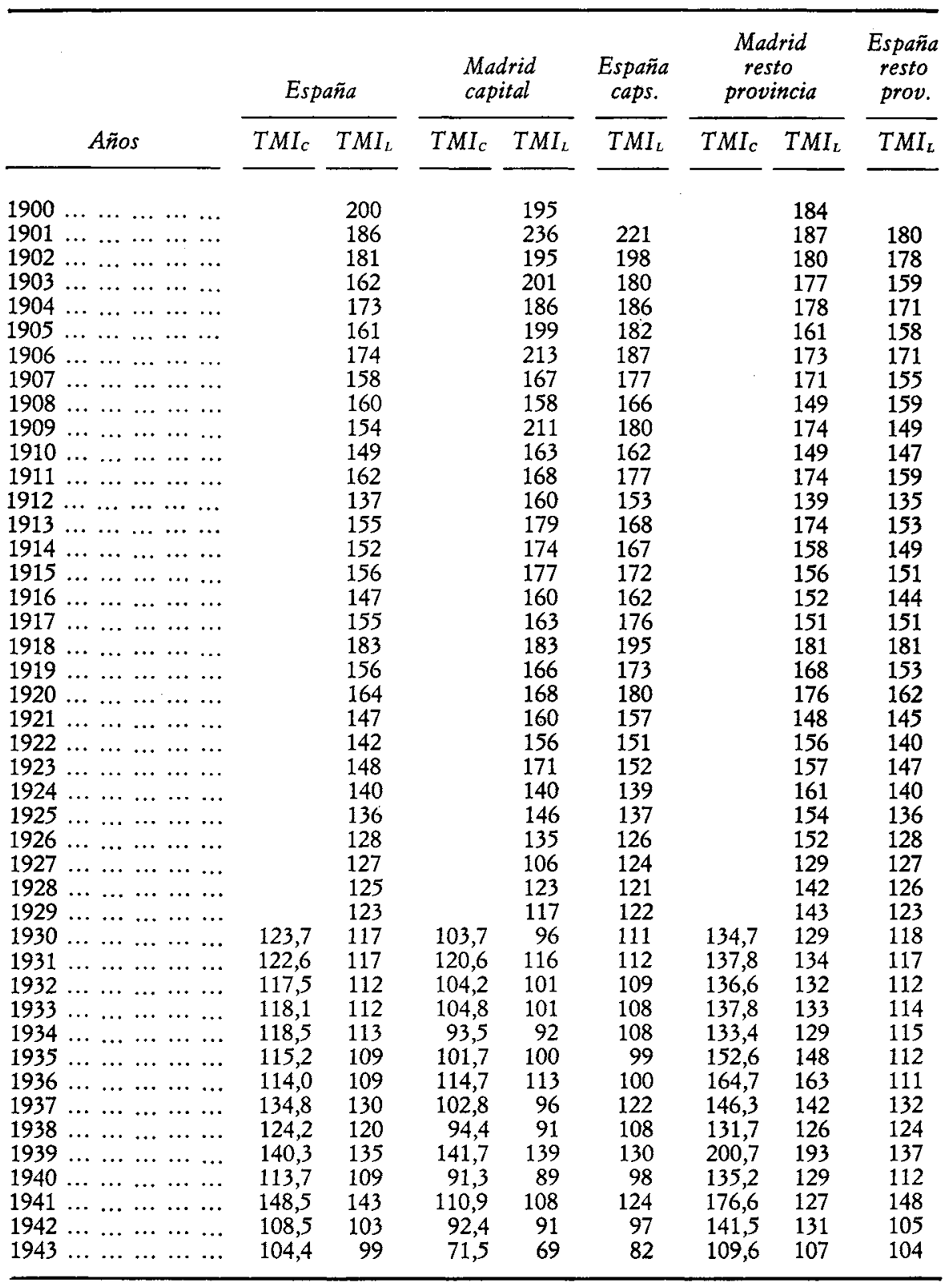




\section{CUADRO 1 (Continuación)}

\section{Tasas de mortalidad infantil corregidas $\left(T M I_{C}\right)(1)$ $y$ legales $\left(T M I_{L}\right)(2), 1900-1970$ \\ Tasas $(1 \times 1.000$ nacidos vivos)}

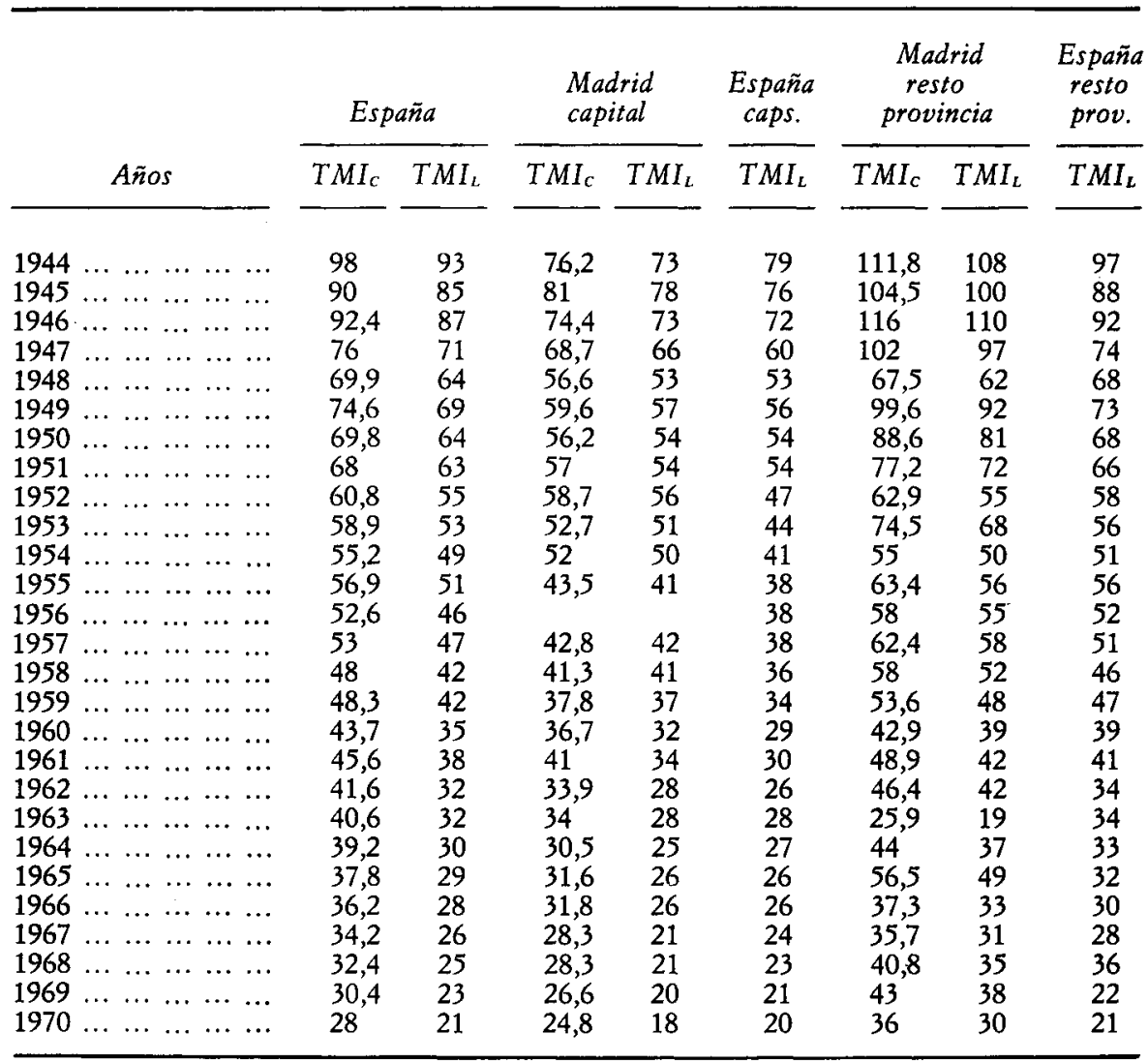

FUENTES: Elaboración propia de las tasas corregidas, así como de las tasas legales a partir de 1943, en base a los Movimientos Naturales de la Población de España. Las tasas legales anteriores a 1943 son de A. ARBelo, La mortalidad de la in. fancia en España, 1900-1950, pp. 315-332.

(1) $T M I_{c}$ : incluye los muertos al nacer y antes de 24 horas como nacidos vivos.

(2) $T M I_{L}$ : no incluye los muertos al nacer y antes de 24 horas como nacidos vivos.

años del mismo; mientras que en el resto de la provincia son superiores para casi la totalidad de los setenta años estudiados.

Por otra parte, Madrid constituye un ámbito de estudio con claras peculiaridades, entre las que destaca los altos niveles de disimilitud entre la mortali- 


\section{GRAFICO 1}

Evolución de la mortalidad infantil (corregida) en Madrid y España, 1930-1970

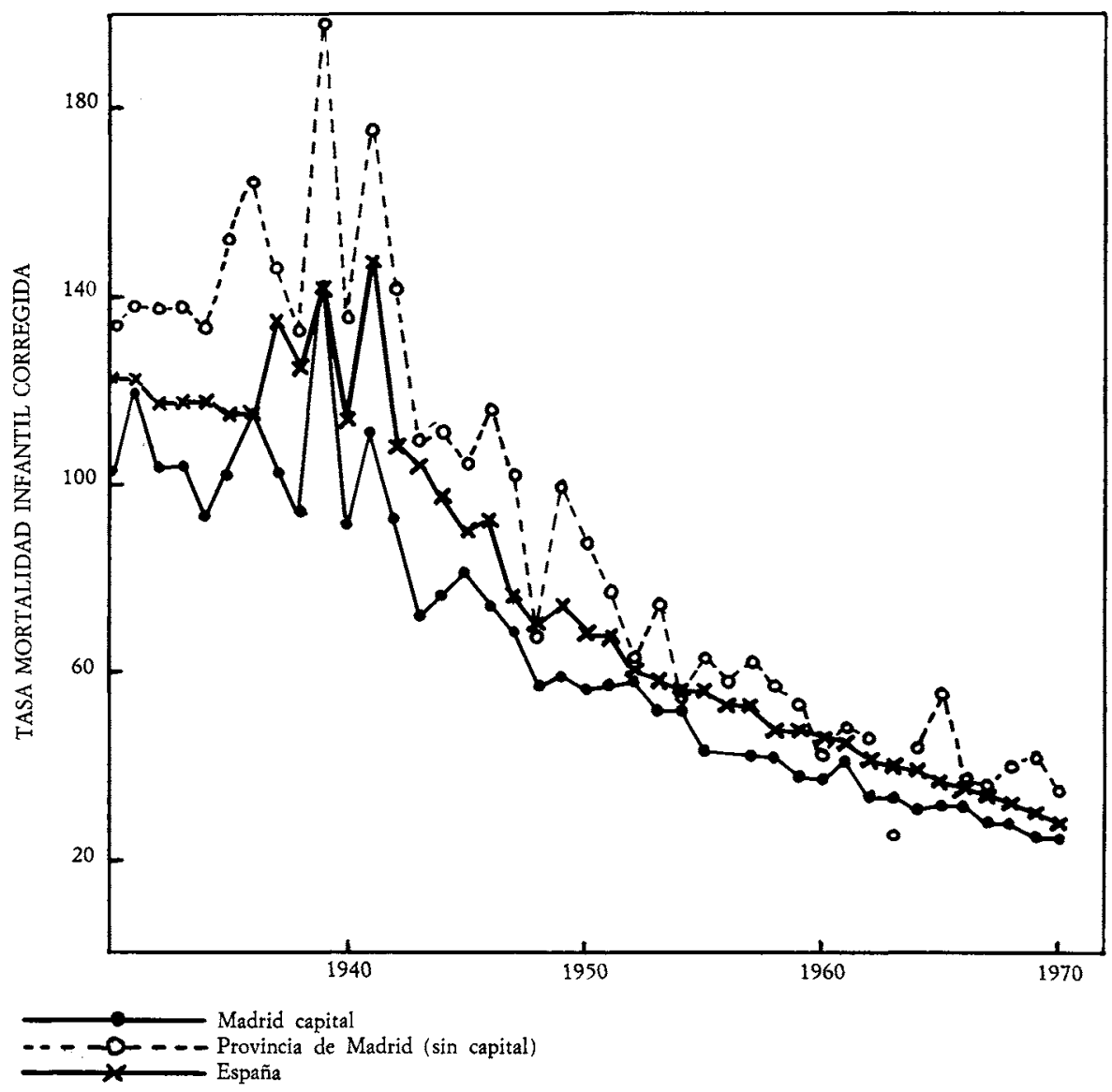

dad infantil de la capital y la del resto de la provincia; estas diferencias son superiores a las existentes entre la media de capitales y la media del resto de las provincias españolas.

A lo largo de los primeros setenta años del siglo, la caída de la mortalidad infantil se produce con variable ritmo y, dentro de su tendencia al descenso, se distinguen dos etapas bien diferenciadas. Durante los primeros cuarenta años del período, el descenso es irregular y lento, y está sometido a continuas fluctuaciones hasta 1921. En el transcurso de esta primera etapa tienen lugar dos crisis demográficas, a las que se dedicará mayor atención más adelante, con impacto sobre la población menor de un año de edad: la gripe de 1918 y la guerra civil de 1936. 
Por el contrario, a partir de los años cuarenta la caída de la mortalidad infantil es rápida y sostenida, y no sufre ninguna interrupción de la tendencia al descenso que sea comparable a las anteriormente citadas.

$\mathrm{Si}$ se excluyen los intervalos de las dos crisis citadas, que interrumpen el descenso de la mortalidad infantil, y se considera el resto del período, se observa que durante los años transcurridos entre $1901-2$ y $1917^{4}$ el descenso, ya comenzado, es aún lento. En efecto, se produce una disminución de las tasas de mortalidad infantil de un 24,4 por 100 en Madrid, que, no obstante, está por encima del logrado en España (19,7 por 100) y el resto de la provincia $(18,6$ por 100$)$.

Una vez superados los efectos de la gripe de 1918, el descenso se reanuda con espectacularidad en Madrid, ya que durante los años transcurridos entre 1921 y 1934 la tasa de mortalidad infantil desciende en la capital en un 41,6 por 100. Este período entre crisis es, sin duda, el de mayor ritmo de descenso para esta ciudad; no así para el total de España $(19,4$ por 100) y el resto de la provincia de Madrid (9,9 por 100).

Posteriormente, una vez finalizada la guerra civil y sus repercusiones, comienza una etapa de descenso irreversible hasta el final del período estudiado. El descenso resulta de gran magnitud para los tres ámbitos que se analizan, ya que alcanza un 77,1 por 100 en Madrid; es similar a la provincia $(74,6$ por 100) y algo menor en el total nacional $(69,7$ por 100). Durante esta segunda etapa se produce el ritmo más rápido de descenso de la mortalidad infantil, tanto en España como en la provincia de Madrid, a diferencia de la capital, que, como se observó, registra su máximo ritmo de caída en los años veinte.

Para cualquiera de los períodos analizados de 1900 a 1970 , la capital madrileña resulta más beneficiada por el descenso de la mortalidad infantil que el resto de la provincia y que España en su conjunto. No es de extrañar que, en las últimas décadas, la caída del fenómeno vaya ralentizándose y que ello ocurra en mayor medida en Madrid capital que en los otros dos ámbitos.

\section{Efecto de las más importantes crisis demográficas del periodo en la mortalidad infantil}

Con anterioridad se ha dicho que el descenso que caracteriza la evolución de la mortalidad en estos setenta años presenta dos interrupciones destacables, ambas en la primera mitad del siglo. La primera es consecuencia de la llegada a España de la pandemia de gripe de 1918; la segunda se debe a la guerra civil iniciada en 1936.

- Para calcular la proporción de descenso de 1901-2 a 1917 se utiliza la tasa media de mortalidad infantil 1901-2, debido a que entre las tasas correspondientes a estos dos primeros años del siglo existe una gran diferencia de nivel atribuible a la aún baja calidad de los datos. 
Desde una perspectiva mundial, la gripe de 1918 ha sido considerada como una de las peores plagas de la historia ${ }^{5}$. Dentro de los niveles alcanzados por esta crisis en Europa —en menor medida que en otras partes del mundo-, España fue muy afectada por la misma. Sin embargo, sus efectos sobre la mortalidad infantil estudiada no reflejan la gravedad aludida. Esta crisis no supera en intensidad a las frecuentes fluctuaciones que a principios de siglo afectaban a Madrid en su aún débil e irregular tendencia al descenso.

La crisis comienza en 1918, pero las repercusiones de la gripe, junto a la influencia que pudiesen tener los factores económico-sociales de la época, la prolongan hasta 1920. Sólo en 1921 las tasas vuelven a los niveles de mortalidad anteriores a la llegada de la citada pandemia. El aumento de la mortalidad del año cumbre de la crisis, 1918, respecto al anterior a su comienzo es de 10,7 por 100 en la capital, 16,6 por 100 en el resto de la provincia y 15,3 por 100 en España.

El virus de la gripe afectó fundamentalmente a jóvenes y adultos y, en mucha menor medida, a niños y ancianos; ésta es la razón de la situación privilegiada de los menores de un año de edad ante dicha pandemia.

Sin duda, la guerra civil, por sus graves consecuencias demográficas, representa la mayor ruptura de la tendencia al descenso en la mortalidad infantil durante el período estudiado, como es evidente en el cuadro 1 . Se ha dicho que la mortalidad infantil es un fenómeno estrechamente dependiente del medio social en el que se produce, lo que explica que la desorganización provocada por una guerra tenga las consecuencias demográficas observadas.

La crisis demográfica inmediata que provoca la guerra civil continúa una vez finalizada ésta en 1939, prolongándose hasta 1941. El incremento de la mortalidad de los menores de un año, desde el año anterior al inicio del conflicto bélico hasta 1939 -año de máxima mortalidad infantil en Madrid-, es de 28,2 por 100 en la capital y 23,9 por 100 en el resto de la provincia, mientras que en España en su conjunto el aumento es menor (17,9 por 100). Por el contrario, aunque los dos ámbitos madrileños sufren un incremento de su mortalidad infantil en 1941, éste no tiene lugar en la medida que en España, donde el aumento es de 22,4 por 100 , por lo que este año es para el total nacional el que peores consecuencias tuvo desde que se inició la crisis.

A lo largo de ambas crisis demográficas, los años de mayor intensidad de las mismas varían cronológicamente entre los tres ámbitos estudiados (gráfico 2); pero, considerando la totalidad del período que abarcó cada crisis, Madrid capital fue afectada en menor medida por la gripe que el conjunto de España, mientras que el resto de la provincia fue la más perjudicada. Por el contrario, las consecuencias de la guerra civil sobre la mortalidad infantil de la provincia fueron superiores a las alcanzadas en la media nacional.

5 A. W. Crosby, Epidemic and Peace, 1918, Westport (Connecticut), Greenwood Press, 1976, p. 315; M. Burnet, Historia de las enfermedades infecciosas, Madrid, Alianza Editorial, 1967, pp. 299-311. 


\section{GRAFICO 2}

Incidencia de la gripe de 1918 y la guerra civil de 1936-39 en la mortalidad infantil en Madrid y España

(Aumento de las tasas de mortalidad infantil respecto a los años base 1902 y 1933 , respectivamente)

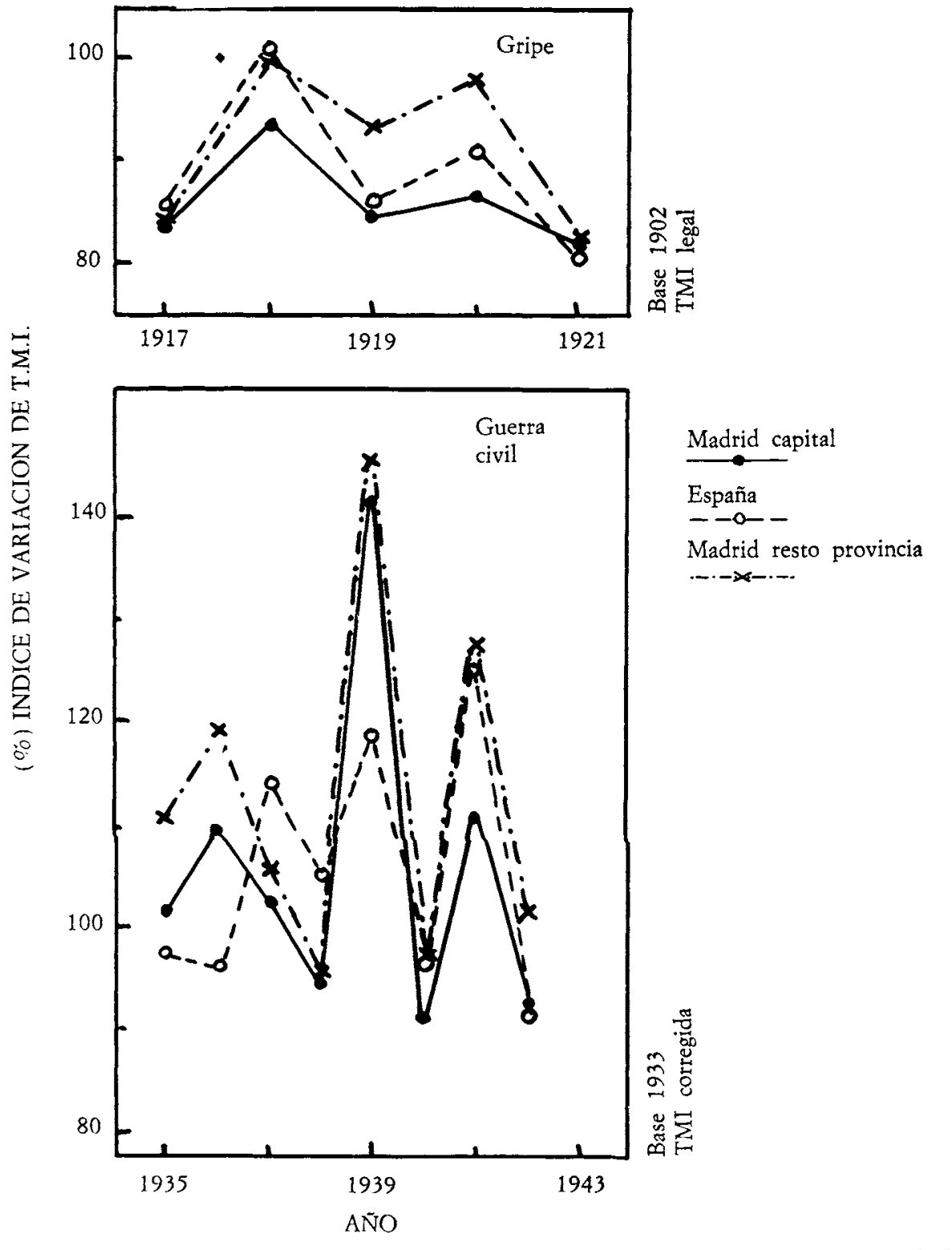


A pesar de la gravedad de estas crisis, que hacen retroceder los niveles de mortalidad infantil en más de una década, sus efectos sólo suponen una interrupción de la clara tendencia al descenso observada, ya que, una vez superados los años que abarcaron, la tendencia se restablece y aún con mayor regularidad.

\section{ALTERACIONES REGISTRALES EN LA EVOLUCION DE LA MORTALIDAD INFANTIL}

Como ocurre no pocas veces, las alteraciones puramente registrales ocultan en parte la evolución real del fenómeno estudiado. Por ello se ha creído oportuno darlas a conocer antes de entrar en la exposición de los importantes cambios observados a lo largo del gradual descenso de la mortalidad infantil.

En primer lugar, es conveniente explicar el motivo por el cual el análisis de este fenómeno demográfico se hace a través de tasas de mortalidad infantil corregidas. Como es bien sabido, el fenómeno de la mortalidad infantil se estudia a través del suceso de defunciones de los menores de un año de edad. La tasa de la mortalidad infantil de un año $t$ será, por tanto, el número de defunciones de los menores de un año de edad en el año $t$ por cada 1.000 nacidos en ese mismo año ${ }^{6}$.

La necesidad de corregir los datos disponibles en España para el cálculo de las tasas se debe a la infravaloración que las cifras de nacidos vivos y de defunciones de menores de un año encierran, y cuyo origen está en el peculiar concepto de «nacido vivo» del Código Civil español (Libro Primero de las personas, tít. $2 .^{\circ}$, cap. $1 .^{\circ}$, art. 30 ), que reza: «para los efectos civiles sólo se reputará nacido el feto que tuviese figura humana y viviere veinticuatro horas enteramente desprendido del seno materno».

Por consiguiente, los muertos al nacer y antes de veinticuatro horas de vida son «criaturas abortivas» (art. 745$)^{7}$, según la ley, y así lo recogen las estadisticas oficiales hasta 1975 , fecha en que, por fin, se identifica el concepto estadístico de nacimiento con el biológico. Hasta entonces es necesario utilizar tasas corregidas para analizar la mortalidad infantil en España y, por ello, la corrección de las tasas consiste en sumar a las defunciones de 0 a 11 meses

- Se adopta esta definición de la mortalidad infantil por ser la utilizada por los organismos internacionales que facilitan información a este respecto: Demograpbic Yearbook, 1982, Naciones Unidas, Nueva York, 1984, Notas Técnicas, p. 62; Annuaire des Statistiques Sanitaires Mondiales 1984, Organización Mundial de la Salud, Ginebra, 1984, cuadro 2 , p. 32 . No obstante, se es consciente de la posibilidad de utilizar tasas que cuantifican con mayor precisión el fenómeno, pero su utilización sólo es necesaria en determinadas circunstancias: W. P. D. LogAN, «Mesure de la mortalité infantile», Bulletin Demographique des Nations Unies, núm. 2, Naciones Unidas, pp. 32-72. También, R. Pressat, El análisis demográfico, Madrid, Fondo de Cultura Económica, 1983, pp. 144-146.

${ }^{7}$ Definición del Movimiento Natural de la Población de España de 1975, Instituto Nacional de Estadística, tomo I, Introducción, p. X. 
cumplidos y a los nacidos vivos las muertes al nacer y de 0 a 23 horas cumplidas.

Al utilizar estos indicadores corregidos se evitan los serios inconvenientes que venían presentando las tasas que llamamos legales ${ }^{8}$, y que son los siguientes. En primer lugar, la falta de homogeneidad entre los conceptos comparados en estudios sincrónicos internacionales que incluyeran a España (lo que también ocurre con otros países), así como en los estudios diacrónicos que abarcasen años anteriores y posteriores a 1975. En segundo lugar, y debido al descenso de la mortalidad infantil durante los años estudiados y a que esa caída es mayor en la mortalidad tardía del primer año de edad, el peso de los muertos durante el primer día de vida aumenta en el total de la muerte infantil, por lo que no considerar su nacimiento y posterior defunción provoca un creciente error. Dicha infravalorización llega a suponer que las tasas legales estén mermadas hasta en más de un cuarto de su valor. Lo expuesto queda patente en el cuadro 2 y los gráficos 3 y 4 , a través de los cuales se ve clara-

\section{CUADRO 2}

Comparación de las tasas de mortalidad infantil corregidas con las legales para Madrid y España, 1930-1970

$\left(I_{D}\right) \cdot 100=$ Indice de diferencia $\left({ }^{*}\right)$ (aumento) de la $T M I_{C}$ respecto a la $T M I_{L}$

\begin{tabular}{|c|c|c|c|c|c|c|c|}
\hline Años & España & $\begin{array}{c}\text { Madrid } \\
\text { capital }\end{array}$ & $\begin{array}{l}\text { Madrid } \\
\text { resto } \\
\text { provincia }\end{array}$ & Años & España & $\begin{array}{c}\text { Madrid } \\
\text { capital }\end{array}$ & $\begin{array}{c}\text { Madrid } \\
\text { resto } \\
\text { provincia }\end{array}$ \\
\hline 1930 & 5,4 & 7,4 & 4,2 & 1951 & 7,3 & 5,2 & 6,7 \\
\hline 1931 & 4,6 & 3,8 & 2,8 & 1952 & 9,5 & 4,6 & 12,6 \\
\hline 1932 & 4,7 & 3,1 & 3,4 & 1953 & 10,0 & 3,2 & 8,7 \\
\hline 1933 & 5,2 & 3,6 & 3,5 & 1954 & 11,2 & 3,8 & 9,1 \\
\hline 1934 & 4,6 & 1,6 & 3,3 & 1955 & 10,4 & 5,7 & 11,7 \\
\hline 1935 & 5,4 & 1,7 & 3,0 & 1956 & 12,5 & & 5,2 \\
\hline 1936 & 4,4 & 1,5 & 1,0 & 1957 & 11,3 & 1,9 & 7,0 \\
\hline 1937 & 3,6 & 6,6 & 2,9 & 1958 & 12,5 & 0,7 & 10,3 \\
\hline 1938 & 3,4 & 3,6 & 4,3 & 1959 & 13,0 & 2,1 & 10,4 \\
\hline 1939 & 3,8 & 1,9 & 3,8 & 1960 & 19,9 & 12,8 & 9,1 \\
\hline 1940 & 4,1 & 2,5 & 4,6 & 1961 & 16,7 & 17,0 & 14,1 \\
\hline 1941 & 3,7 & 2,6 & & 1962 & 23,0 & 17,4 & 9,5 \\
\hline 1942 & 5,1 & 1,5 & 7,4 & 1963 & 21,2 & 17,6 & \\
\hline 1943 & 5,2 & 3,5 & 2,4 & 1964 & 23,5 & 18,0 & 15,9 \\
\hline 1944 & 5,1 & 4,2 & 3,4 & 1965 & 23,3 & 17,7 & 13,3 \\
\hline 1945 & 5,6 & 3,7 & 4,3 & 1966 & 22,6 & 18,2 & 11,5 \\
\hline 1946 & 5,8 & 1,9 & 5,2 & 1967 & 24,0 & 25,8 & 13,2 \\
\hline 1947 & 6,6 & 3,9 & 4,9 & 1968 & 22,8 & 25,8 & 14,2 \\
\hline 1948 & 8,4 & 6,4 & 8,1 & 1969 & 24,3 & 24,8 & 11,6 \\
\hline 1949 & 7,5 & 4,4 & 7,6 & 1970 & 25,0 & 27,4 & 16,7 \\
\hline 1950 & 8,3 & 3,9 & 8,6 & & & & \\
\hline
\end{tabular}

(*) $I_{D} \cdot 100=100-\left(\frac{T M I_{L}}{T M I_{C}} \cdot 100\right) \quad \begin{aligned} & T M I_{L}=\text { tasa de mortalidad infantil legal } \\ & T M I_{C}=\text { tasa de mortalidad infantil corregida }\end{aligned}$

- Término utilizado por A. Arbelo en Demografía sanitaria, op. cit., p. 117. Se refiere a las tasas sin corregir. 


\section{GRAFICO 3}

Evolución del error de medición de las tasas legales respecto a las corregidas en España, 1930-1970

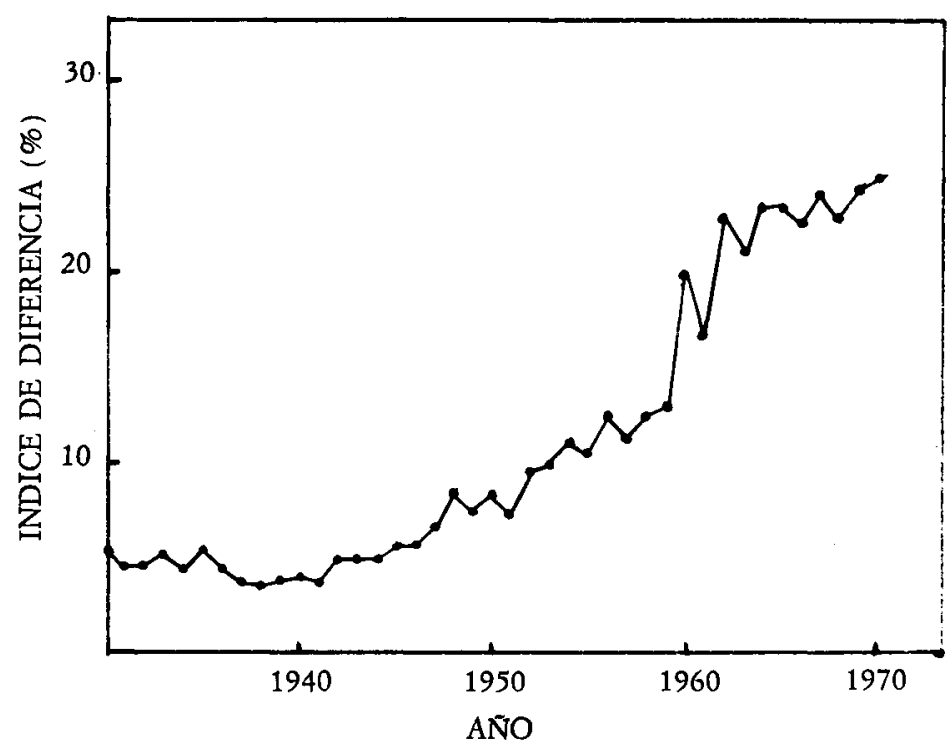

mente la necesidad de corregir este tradicional error, debido al progresivo distanciamiento de ambos tipos de tasas.

Por todo ello, se ha elaborado la serie de tasas corregidas a partir de 1930, año en que comienzan a publicarse ininterrumpidamente los datos necesarios para el cálculo de las mismas ${ }^{9}$.

Además, existen dos obstáculos estadísticos que interfieren especialmente en el estudio del proceso seguido por la mortalidad infantil. Por una parte, está el hecho de que las defunciones se registren en base al lugar del fallecimiento en vez del de la residencia materna, lo que, unido a la centralización hospitalaria en las grandes ciudades, provoca una hipervaloración creciente de la mortalidad infantil -especialmente la precoz- en las capitales de provincia a medida que se generaliza el parto hospitalario en España.

9 Las tasas de mortalidad infantil corregidas para el total de España (sin desagregación provincial) han sido calculadas por otros autores para períodos breves. Entre otros, A. ARBELo, Demografía sanitaria, op. cit. (de 1950 a 1970), p. 500, y A. DE Miguel, La pirámide social española, Madrid, Fundación Juan March, Ariel, 1977 (de 1960 a 1973), p. 29. 


\section{GRAFICO 4}

Proporción del error de medición de las tasas legales

frente a las corregidas (\%) en 1931 v 1970

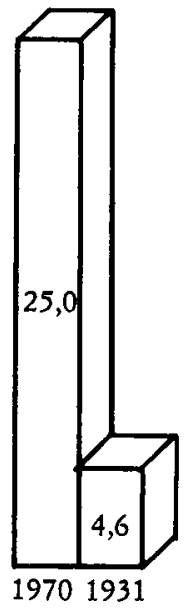

España
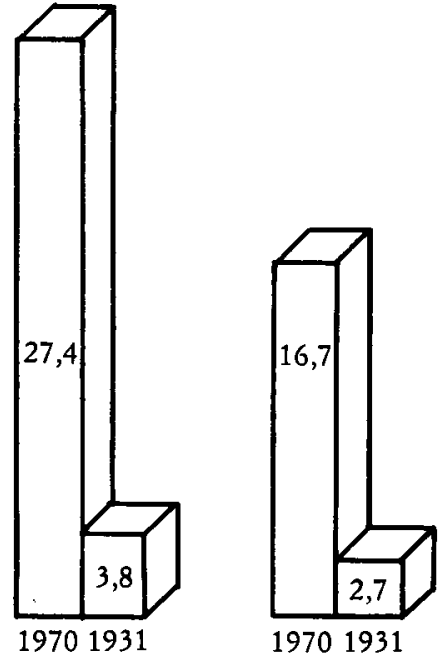

Madrid capital Madrid resto provincia

Al principio del período, la hipervaloración de la mortalidad infantil en las ciudades pudo deberse en parte a la localización en las mismas de las inclusas, ya que en estos centros la mortalidad estaba muy por encima de la del resto de la sociedad ${ }^{10}$.

Por otra parte, la artificiosidad del concepto legal de «nacido vivo», al que ya se ha hecho referencia, ha dado lugar a un subregistro de los muertos con menos de un día de vida - considerados legalmente abortos- (véase apartado 3).

Este error parece corregirse con la normalización de los modelos de «partes de alumbramiento de criaturas abortivas» y los «cuestionarios para la declaración de nacimiento en el registro civil», cuya utilización se generaliza en 1960. En ellos se exige precisión en el período de vida del fallecido y se

$10 \mathrm{~V}$. Fernández Vargas y L. Navarro, «La desigualdad ante la muerte. Presentación de una investigación sobre Madrid», Revista Internacional de Sociología, núm. 47, julio-septiembre 1983, pp. 441-445. 
recuerda el requisito de haber vivido veinticuatro horas para considerar a una criatura «nacida viva» ${ }^{11}$.

Esta es la razón por la que se observa un brusco aumento del índice de diferencia entre las tasas legales y las tasas corregidas de mortalidad infantil (cuadro 2 y gráfico 3) en el curso de 1959 a 1960, y el hecho de que Madrid capital reciba mortalidad infantil del resto de la provincia y de las provincias limítrofes en sus centros hospitalarios explica el que sus índices sean aún más elevados.

Además de los problemas, tanto de tipo legal como puramente estadísticos, que se acaban de exponer, se han observado irregularidades en los datos oficiales que inducen a dudar de la calidad de los mismos; esto se hace más evidente cuanto más desagregados estén los datos. Así, la evolución de la provincia de Madrid (excluida la capital) es muy irregular, lo que no encuentra un paralelo en las provincias españolas (excluidas las capitales) en su conjunto. Ello puede deberse a que las tasas nacionales, por ser valores medios, siempre presentan una mayor estabilidad. Este problema surgirá en el ámbito provincial frecuentemente, sea cual fuere el criterio de la desagregación.

Asimismo, se ha de tener en cuenta la existencia de un subregistro de sucesos demográficos infantiles ${ }^{12}$, el cual habrá ido disminuyendo a medida que aumentaba la valoración de la vida del niño. En el caso de las defunciones, dicho subregistro es previsible que fuese de mayor magnitud cuanto más próximas al nacimiento ocurriesen éstas; por ello, la aludida disminución del error deficitario en el registro ha podido reflejarse en un aumento de las submortalidades ${ }^{13}$ infantiles más tempranas, que al ser analizadas a través de tasas corregidas son medidas con mayor precisión. También es muy probable que este subregistro desapareciese con anterioridad en las capitales, pero ello está insuficientemente estudiado, tanto en España como en Europa.

A pesar de las numerosas alteraciones registrales expuestas, a través del estudio del descenso de la mortalidad infantil se han deducido interesantes conclusiones que arrojan luz sobre tan trascendental proceso en Madrid.

"La normalización de estos «modelos» se debe a la puesta en vigor de la Orden del Ministerio de Justicia de fecha 24 de diciembre de 1958. Con anterioridad, los partes y declaraciones podían extenderse en papel común (Orden del 27-1-1932, Ministerio de Justicia), de manera que la arbitrariedad del facultativo hacía imposible una mayor calidad del registro.

12 Algunos de los autores que han constatado subregistro en los sucesos demográficos infantiles son A. G. Barbanc h o y M. Delgado-Cabeza, «Les erreurs sur l'âge de la population infantile dans les recensements espagnols», Population, núms. 4-5, julio-octubre 1984, pp. 874-883; W. LEASURE, «Factors involved in the decline of fertility in Spain, 1900-1950», Population Studies, XVI, núm. 3, 1963, p. 271; M. Livi BaccI, «Fertility and Nupciality changes in Spain from the late 18th to the early 20th centurym, Population Studies, XXII, núm. 2, 1968, pp. 232-233.

${ }^{13}$ El término submortalidad se utiliza en su acepción de una parte de la mortalidad infantil total. 


\section{EVOLUCION DE LOS COMPONENTES DE LA MORTALIDAD INFANTIL DESDE DIFERENTES PERSPECTIVAS Y SUS CAMBIOS}

\section{Mortalidad diferencial según el criterio ecológico}

El espectacular descenso de la mortalidad infantil ocurre al mismo tiempo que notables cambios en la sociedad en que se producen esos fallecimientos. A principios del período, la inadecuada alimentación del lactante y las malas condiciones higiénico-sanitarias determinaban que las frecuentes invasiones de enfermedades infecciosas, endémicas o no, encontrasen fácil presa en seres desnutridos ${ }^{14}$, lo que fundamentalmente justifica las altas tasas de mortalidad infantil de la época.

Este panorama era crítica en las ciudades, y especialmente en Madrid, donde el crecimiento constante, debido en buena parte a la inmigración, provocaba el hacinamiento de la población en una ciudad que apenas se expandía territorialmente y que carecía de una política higiénico-sanitaria ${ }^{15}$.

Por este motivo, resulta lógico que Madrid capital y el conjunto de las capitales españolas superasen en la mortalidad de los menores de un año de edad al resto de la nación. Las peores condiciones de vida en las ciudades eran el origen de la mortalidad diferencial observada desde un criterio ecológico, pero al equipararse las condiciones de vida de las capitales con las del resto de la población las tasas se igualaron. En Madrid esto ocurrió en 1924 y, posteriormente —en 1926-, en el conjunto de España. Desde entonces, la sobremortalidad existente con anterioridad en las capitales se encuentra en el resto de las provincias.

Quizá sea la capital, Madrid, una de las ciudades que más cambia en sus condiciones de vida en el período analizado. Por ello, a partir del cambio señalado y durante el resto del período, se mantienen entre Madrid capital y el resto de la provincia diferencias en el nivel de mortalidad infantil superiores a las encontradas en España.

El cambio ecológico-demográfico a que se acaba de hacer referencia es, cronológicamente, el primer cambio destacable de los que se producen en la mortalidad infantil a lo largo de los años estudiados.

La diferencia existente entre Madrid capital y el resto de la provincia demuestra que, efectivamente, se trata de dos marcos sociodemográficos muy distintos y que, a pesar del teórico papel de la capital como núcleo difusor de condiciones de vida sobre el resto de la provincia, ello no ha dado lugar a que se igualasen los niveles respectivos de la mortalidad infantil.

${ }^{14}$ Recuérdese la relación entre el estado nutritivo y la resistencia inmunológica del huésped, magníficamente tratada por Th. MACKEOwN, op. cit., pp. 157-174.

${ }_{13}$ Situación descrita en el valioso estudio de Ph. Hauser, Madrid bajo el punto de vista médico-social, Madrid, Editora Nacional, 1902, vol. I, pp. 179-334. 


\section{Mortalidad diferencial según la etiologia del fallecimiento}

Después de analizar el descenso del fenómeno demográfico estudiado, surge la necesidad de conocer qué tipo de mortalidad infantil es el causante principal de este deșcenso. A estos efectos conviene clasificar la mortalidad infantil en dos grandes grupos, según el criterio de la causa última de la defunción: causas de origen exógeno (enfermedades infecciosas, desnutrición o alimentación inadecuada y accidentes) y causas de origen endógeno (inmadurez del nacido, malformación y debilidad congénitas y traumatismos del parto).

Para analizar la mortalidad infantil según su etiología se ha utilizado el método que parece ser más objetivo y fiable en un país que no cuenta con muy buenas estadísticas por causas de muerte: el método biométrico de Bourgeois-Pichat ${ }^{16}$, cuya validez ha sido contrastada positivamente en numerosas poblaciones.

El método Bourgeois-Pichat permite dividir la mortalidad infantil en sus dos componentes por etiología (mortalidad endógena y mortalidad exógena) a partir del conocimiento de los fallecidos de menos de un año por edad en días. Este método se basa en el hallazgo por Bourgeois-Pichat de la función que relaciona las defunciones a una edad fisiológica determinada con esa edad.

Según el autor, las defunciones por causas exógenas son proporcionales al cubo de la edad fisiológica; por tanto:

$$
D(x)=a+b \log ^{3}(x+1)
$$

siendo:

$$
\begin{aligned}
& D(x): \text { la serie acumulada de fallecidos } \\
& x: \text { edad en días } \\
& a: \text { fallecidos endógenos. }
\end{aligned}
$$

En el método expuesto se parte de que los fallecimientos endógenos son despreciables a partir del primer mes de vida y, por ello, a partir de los treinta días de vida se calcula la serie acumulada de defunciones; serie que aumenta sólo por el efecto de los fallecimientos exógenos.

Por tanto, la función es un ajuste lineal de defunciones (entre uno y once

16 J. Bourgeois-Pichat, «La mesure de la mortalité infantile. I. Principes et méthodes», Population, núm. 2, 1951, pp. 233-248, y «La mesure de la mortalité infantile. II. Les causes de déces», Population, núm. 3, 1951, pp. 459-480. Este método ha sido utilizado para España y para períodos muy cortos: J. Villar Salinas, «La mortalidad infantil contemporánea de las provincias españolas disociada en sus dos grandes causas», Revista Internacional de Sociología, núm. 34, abril-junio 1951 (calcula la media del período 1944-1948), pp. 365-388; J. Leguina, «La mortalidad infantil. Aplicación del método biométrico de Bourgeois-Pichat a las provincias españolas», Estadística Española, núm. 47, abril-junio 1970 (de 1960 a 1965), pp. 95-114. 
meses) acumuladas en función de la edad (ajuste realizado por mínimos cuadros).

El análisis de la mortalidad infantil según su etiología presenta la ventaja de reflejar con nitidez el peso de la evolución del marco social en el descenso de las muertes infantiles, debido a la estrecha relación existente entre las causas de muerte exógenas y el medio en el que se producen.

Es evidente que, como a cualquier clasificación, a la propuesta (causa exógena y endógena) pueden hacérsele objeciones. Ciertamente, en la muerte confluyen factores biológicos y ambientales difíciles de aislar y, por ello, la frontera entre ambas etiologías no es precisa ni estable, ya que la misma puede ir evolucionando a medida que avanza la medicina infantil. Sin embargo, a través del método de Bourgeois-Pichat se evitan los frecuentes errores de diagnóstico que afectan a las estadísticas por causas específicas de muerte, especialmente allí donde no pueda confiarse en la calidad de los datos disponibles.

En España no se dispone de los datos necesarios para la aplicación del método citado (defunciones de menores de un año de edad desagregados por períodos de vida) hasta 1941. Aunque estos datos, muy probablemente, no reúnen una mínima calidad hasta 1945, ya que durante esos primeros cuatro años los erróneos resultados obtenidos de la aplicación del método así lo indican, puesto que no guardan ninguna coherencia con los obtenidos durante el resto del período.

Del análisis de la mortalidad infantil según la etiología del fallecimiento se deduce que el descenso de la mortalidad infantil se debe a la caída de la mortalidad por causa exógena, disminuyendo la misma hasta en un 85,7 por 100 en la capital (cuadro 3).

En este caso, como en otros muchos, Madrid capital y España se asemejan en el proceso seguido, ya que el descenso en todo el país es de un 84,2 por 100. En ambos medios, los períodos con mayor ritmo de descenso en las muertes causadas por esta etiología son los últimos cinco años de los setenta analizados, además del quinquenio 1945-1950, en Madrid, y el de 1955-1960, en España (cuadro 4). En cuanto al resto de la provincia de Madrid, debido a la dudosa calidad de los datos desagregados, ya citada, y a la irregularidad que presentan sus indicadores según etiología, es preferible prescindir en esta ocasión de su comparación con los otros dos ámbitos.

La contribución de la mortalidad por causa endógena a ese descenso de la mortalidad infantil durante ese mismo período es negativa, ya que no sólo no disminuye, sino que aumenta su nivel. No obstante, es tan baja la proporción que representa en la mortalidad infantil global que este incremento no se refleja en el total de la misma. En este aumento de la mortalidad por causa endógena han de distinguirse dos tipos de explicaciones. La primera y más importante es que este aumento no se debe tomar como una tendencia para- 


\section{CUADRO 3}

Tasas de mortalidad infantil según la etiologia del fallecimiento (Madrid capital)

\begin{tabular}{|c|c|c|c|c|c|}
\hline \multirow[b]{2}{*}{$A \tilde{n} o s\left(^{*}\right)$} & \multicolumn{3}{|c|}{ Tasas corregidas $(1 \times 1.000)$} & \multicolumn{2}{|c|}{$(1 \times 100)$ de $1 \mathrm{a} T M I$} \\
\hline & $\begin{array}{c}T M I \\
\text { total } \\
(1)\end{array}$ & $\begin{array}{c}T M I \\
\text { endógena } \\
\text { (2) }\end{array}$ & $\begin{array}{c}T M I \\
\text { exógena } \\
(3)\end{array}$ & $\begin{array}{l}\text { Mortalidad } \\
\text { endógena } \\
\quad(2 / 1)\end{array}$ & $\begin{array}{c}\text { Mortalidad } \\
\text { exógena } \\
(3 / 1)\end{array}$ \\
\hline 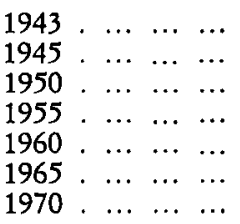 & $\begin{array}{l}71,5 \\
77,2 \\
56,2 \\
43,5 \\
36,7 \\
32,5 \\
24,8\end{array}$ & $\begin{array}{r}5,2 \\
3,8 \\
5,9 \\
7,6 \\
11,1 \\
14,8 \\
14,3\end{array}$ & $\begin{array}{l}66,3 \\
73,4 \\
50,3 \\
35,9 \\
25,6 \\
17,7 \\
10,5\end{array}$ & $\begin{array}{r}7,3 \\
4,9 \\
10,5 \\
17,5 \\
30,2 \\
45,5 \\
57,7\end{array}$ & $\begin{array}{l}92,7 \\
95,1 \\
89,5 \\
82,5 \\
69,8 \\
54,5 \\
42,3\end{array}$ \\
\hline
\end{tabular}

(*) 1941 y 1942 no aplicable el método utilizado por irregularidades en los datos.

FuENTE: Elaboración propia en base a los MNP (INE).

\section{CUADRO 4}

Tasas de mortalidad infantil según la etiologia del fallecimiento (España)

\begin{tabular}{|c|c|c|c|c|c|}
\hline \multirow[b]{2}{*}{$A \tilde{n} o s(*)$} & \multicolumn{3}{|c|}{ Tasas $1 \times 1.000$} & \multicolumn{2}{|c|}{$1 \times 100$ de la $T M I$} \\
\hline & $\begin{array}{c}T M I \\
\text { total } \\
(1)\end{array}$ & $\begin{array}{c}T M I \\
\text { endógena } \\
\text { (2) }\end{array}$ & $\begin{array}{c}T M I \\
\text { exógena } \\
\text { (3) }\end{array}$ & $\begin{array}{c}\text { Mortalidad } \\
\text { endógena } \\
(2 / 1)\end{array}$ & $\begin{array}{c}\text { Mortalidad } \\
\text { exógena } \\
(3 / 1)\end{array}$ \\
\hline $\begin{array}{lll}\ldots & \ldots & \ldots\end{array}$ & 108,5 & 9,7 & 98,8 & 8,9 & 91,1 \\
\hline $1943 \ldots \ldots \ldots \ldots$ & 104,4 & 9,9 & 94,5 & 9,5 & 90,5 \\
\hline $1945, \ldots \ldots \ldots$ & 90 & 11 & 79 & 12,2 & 87,8 \\
\hline $1950 \ldots \ldots \ldots$ & 69,8 & 10,8 & 59 & 15,5 & 84,5 \\
\hline $1955 \ldots \ldots \ldots$ & 56,9 & 12,6 & 44,3 & 22,1 & 77,9 \\
\hline $1960 \ldots \ldots \ldots$ & 43,7 & 14,7 & 29 & 33,6 & 66,4 \\
\hline $1965, \ldots \ldots \ldots$ & 37,8 & 16,2 & 21,6 & 42,9 & 57,1 \\
\hline $1970 \ldots \ldots \ldots$ & 28 & 15,5 & 12,5 & 55,4 & 44,6 \\
\hline
\end{tabular}

(*) 1941 no aplicable.

Fuente: Elaboración propia en base a los MNP (INE). 


\section{CUADRO 5}

Tasas de mortalidad infantil según la etiología del fallecimiento (Madrid provincia)

\begin{tabular}{|c|c|c|c|c|c|}
\hline \multirow[b]{2}{*}{$A \tilde{n} o s(*)$} & \multicolumn{3}{|c|}{ Tasas $1 \times 1.000$} & \multicolumn{2}{|c|}{$1 \times 100$ de la $T M I$} \\
\hline & $\begin{array}{c}T M I \\
\text { total } \\
(1)\end{array}$ & $\begin{array}{c}\text { TMI } \\
\text { endógena } \\
\text { (2) }\end{array}$ & $\begin{array}{l}\text { TMI } \\
\text { exógena } \\
(3)\end{array}$ & $\begin{array}{l}\text { Mortalidad } \\
\text { endógena } \\
(2 / 1)\end{array}$ & $\begin{array}{c}\text { Mortalidad } \\
\text { exógena } \\
(3 / 1)\end{array}$ \\
\hline $1943 \ldots \ldots \ldots$ & 82,3 & 4,5 & 77,8 & 5,5 & 94,5 \\
\hline $1950 \ldots \ldots \ldots$ & 63,8 & 6,3 & 57,5 & 9,9 & 90,1 \\
\hline $1955 \cdot \ldots \ldots \ldots$ & 46 & 8,6 & 37,4 & 18,7 & 81,3 \\
\hline $1960 \ldots \ldots \ldots$ & 37,3 & 10,7 & 26,6 & 28,7 & 71,3 \\
\hline $1965 \ldots \ldots \ldots$ & 33,3 & 14,8 & 18,5 & 44,4 & 55,6 \\
\hline $1970 \ldots \ldots \ldots$ & 25,2 & 14 & 11,2 & 55,6 & 44,4 \\
\hline
\end{tabular}

(*) No aplicable: 1941,42 y 45 .

FueNTE: Elaboración propia en base a los MNP (INE).

\section{CUADRO 6}

Tasas de mortalidad infantil según la etiología del fallecimiento (Madrid resto provincia)

\begin{tabular}{|c|c|c|c|c|c|}
\hline \multirow[b]{2}{*}{$A \operatorname{nos}(*)$} & \multicolumn{3}{|c|}{ Tasas $1 \times 1.000$} & \multicolumn{2}{|c|}{$1 \times 100$ de la TMI } \\
\hline & $\begin{array}{c}T M I \\
\text { total } \\
(1)\end{array}$ & $\begin{array}{c}\text { TMI } \\
\text { endógena } \\
(2)\end{array}$ & $\begin{array}{l}T M I \\
\text { exógena } \\
\text { (3) }\end{array}$ & $\begin{array}{c}\text { Mortalidad } \\
\text { endógena } \\
(2 / 1)\end{array}$ & $\begin{array}{c}\text { Mortalidad } \\
\text { exógena } \\
(3 / 1)\end{array}$ \\
\hline $1955 . \ldots \ldots \ldots$ & 63,4 & 16,3 & 47,1 & 25,7 & 74,3 \\
\hline $1960 \ldots \ldots \ldots$ & 42,9 & 7,2 & 35,7 & 16,8 & 83,2 \\
\hline $1965 \ldots \ldots \ldots$ & 44,3 & 13,9 & 30,4 & 31,4 & 68,6 \\
\hline $1970 \ldots \ldots \ldots$ & 36 & 6,8 & 29,2 & 18,9 & 81,1 \\
\hline
\end{tabular}

(*) No aplicable: $1941,42,43,45$ y 50 .

FUENTE: Elaboración propia en base a los MNP (INE). 
lela al descenso de la mortalidad exógena, sino que, al contrario, por la experiencia que se tiene de estudios realizados en otros países europeos que cuentan con datos fiables ${ }^{17}$ incluso para períodos anteriores al analizado, se sabe que la mortalidad endógena tiene una tendencia de descenso que, aunque lenta, suele ser anterior al comienzo de la caída de la mortalidad exógena.

Asimismo, la mortalidad infantil endógena se halla sometida a lo largo de su evolución a continuas oscilaciones, por lo que la presente no deja de ser una más de las que la caracterizan ${ }^{18}$, coincidiendo sus momentos de ascensión y estabilización con el período estudiado.

No obstante, se debe considerar la influencia que ha podido tener en el aumento de esta submortalidad la paulatina disminución del subregistro de defunciones existente con anterioridad, y al que ya se ha hecho referencia, probablemente mayor cuanto más cercano al nacimiento ocurriese el suceso. Tampoco se debe olvidar que el registro de defunciones según el lugar del deceso provoca un aumento de la mortalidad endógena mayor en las capitales, y especialmente en Madrid, por las razones expuestas en el apartado 2.

Sin embargo, a pesar de estas alteraciones registrales, este incremento de causas endógenas de muerte puede deberse en parte a un efecto contradictorio de los avances científico-médicos frente a la mortalidad. El aumento podría ser consecuencia de que, a partir de mediados de los años cincuenta, se hagan posibles y lleven a término embarazos que con anterioridad hubiesen sido inviables, lo que en buena medida podría evitar un proceso selectivo que aumente la mortalidad por causa endógena.

De lo expuesto se desprende que el origen del descenso de la mortalidad infantil se debe a la caída de las causas exógenas de muerte (gráfico 5). Esta etiología representaba el 95,1 por 100 de las muertes infantiles en 1945, y en 1970 sólo el 42,3 por 100, en el caso de Madrid capital.

En España, donde la mortalidad por causas exógenas en 1945 representaba el 87,8 por 100 , en 1970 cae hasta el 44,6 por 100 del total de muertes de menores de un año de edad. Entre 1965 y 1970 ambas submortalidades descienden conjuntamente, lo que podría suponer el final del aumento endógeno, es decir, el cierre de la oscilación del período estudiado, pero la comprobación de este supuesto implicaría investigar un período posterior.

Esta importante caída de las tasas de mortalidad por causa exógena hace que ésta deje de representar la proporción mayoritaria de defunciones en el total de la mortalidad de los menores de un año. Este proceso conduce al segundo y más importante de los cambios destacables de la evolución de la

${ }^{17}$ M. Poulain y D. Tabutin, «La mortalité aux jeunes âges en Europe et en Amérique du Nord du xix à nos jours», en P. M. Boulanger y D. Tabutin (eds.), La mortalité des enfants dans le monde et dans l'bistoire, Universidad Católica de Lovaina, Lieja, Ordina Editions, 1980, pp. 129-133.

${ }_{18}$ Oscilaciones que han sido observadas (aunque más breves) en algunos países de Europa y América del Norte por J. BouRgeOIS-PIC HAT, «Evolution recente de la mortalité infantile», Population, núm. 3, junio-julio 1964, pp. 417-438. 


\section{GRAFICO 5}

Evolución de la mortalidad infantil según la etiología del fallecimiento, 1945-1970

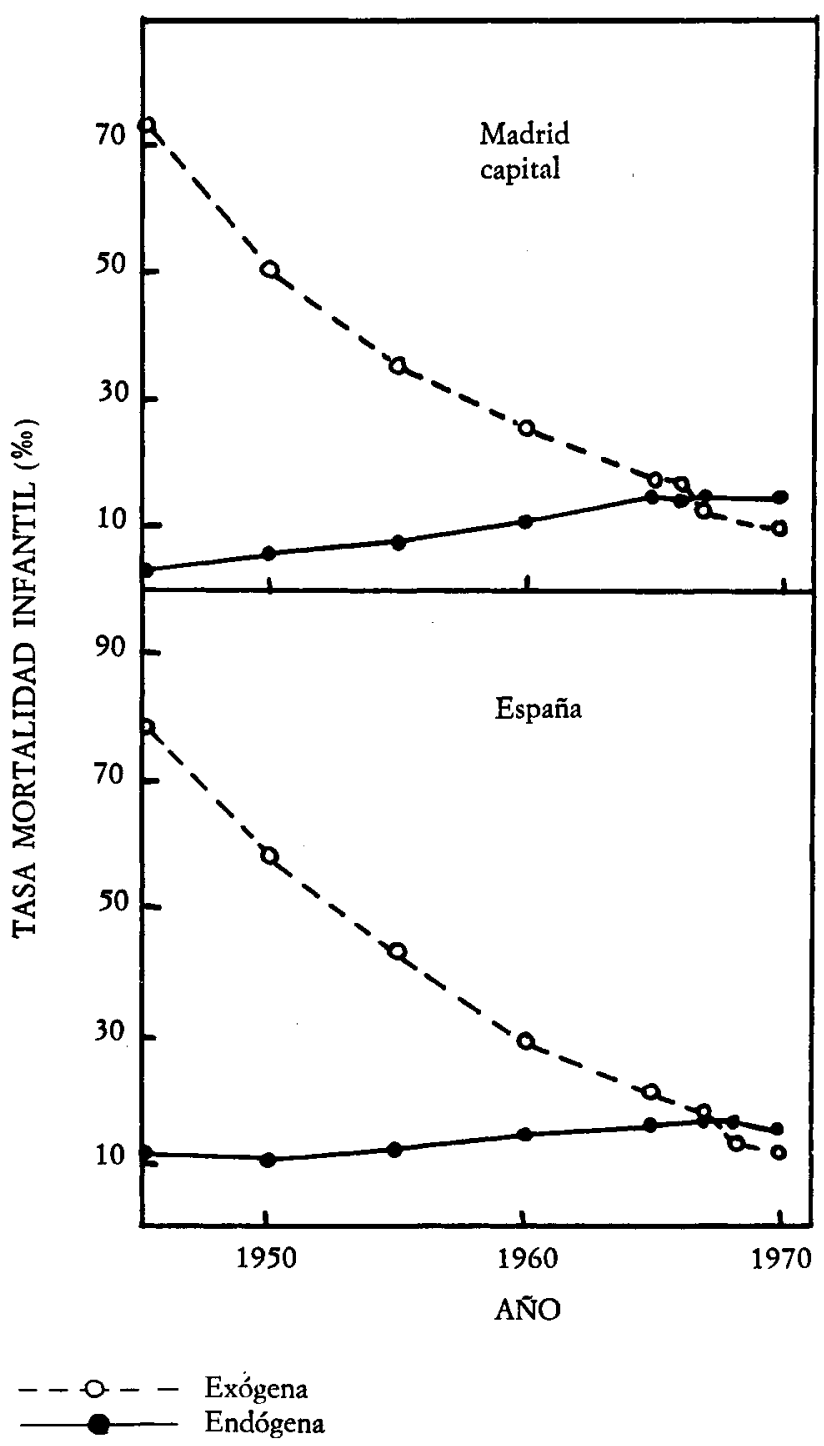


mortalidad infantil en Madrid en los setenta primeros años del siglo y, dada la similitud del proceso, también en España: las tasas de la mortalidad por causa exógena, tradicionalmente mucho más altas que las endógenas, se igualan en 1967, fecha en la que estas últimas empiezan a ser superiores, representando desde entonces la mortalidad exógena menos - y cada vez menosdel 50 por 100 de la mortalidad infantil.

Detrás de esta evolución de la mortalidad infantil según la etiología del fallecimiento está el desarrollo de una sociedad que en sólo veinticinco años elevó notablemente su nivel económico, cultural y médico-sanitario. Si en la provincia de Madrid (ya que no en la totalidad de España) en 1945 habían comenzado a disminuir las defunciones derivadas de una inadecuada alimentación (cantidad, digeribilidad y dosificación), continuaban, sin embargo, las malas condiciones de habitabilidad de los hogares, el bajo nivel cultural de la población (de especial importancia el de las madres) y la inexistencia de una medicina preventiva, junto a unos servicios de puericultura y de pediatría incipientes. Todos estos factores influyentes en la mortalidad infantil justifican que para esa fecha continúe siendo la mortalidad dominante la causada por enfermedades de tipo exógeno.

El marco social descrito fue cambiando al coincidir con una etapa de desarrollo económico. Este desarrollo, si bien no puede afirmarse que fuese la causa directa del descenso de la mortalidad infantil, posibilitó (a través de los factores citados) el que se mantuviesen las disminuciones conseguidas y que un aumento del nivel de vida en su más amplia significación repercutiera en las probabilidades de supervivencia de los menores de un año.

Sin embargo, si durante la primera mitad del siglo la mejora alimenticia y el incremento de las condiciones higiénicas pudieron ser determinantes, durante la segunda mitad, sin restar importancia a estas variables, la rapidez del descenso hay que buscarla en un acontecimiento de gran trascendencia: la llegada y empleo de antibióticos en España, así como la generalización del uso de las sulfamidas. Esto hizo que las enfermedades infecciosas, primera causa de muerte de los menores de un año de edad, pudieran ser curadas con mayor facilidad.

Si a través de la evolución de las tasas de mortalidad infantil exógena se va reflejando el cambio de la sociedad en la que tiene lugar este fenómeno, de cara al futuro la mortalidad por causa endógena, por su origen fundamentalmente genético (salvo en la cuestionable inclusión en esta etiología de los traumatismos del parto), va a medir la evolución de los conocimientos biomédicos al respecto y su aplicación.

En la caída de las causas exógenas de muerte y su sustitución por las causas endógenas como etiología dominante en la mortalidad infantil (gráfico 6) se basa el hecho demográfico por excelencia del período estudiado, el descenso de la mortalidad infantil, y de aquél se derivan la mayoría de los cambios observables en este objeto de estudio durante el período analizado. 


\section{GRAFICO 6}

Evolución de la proporción representada por la mortalidad endógena en la mortalidad infantil, 1945-1970

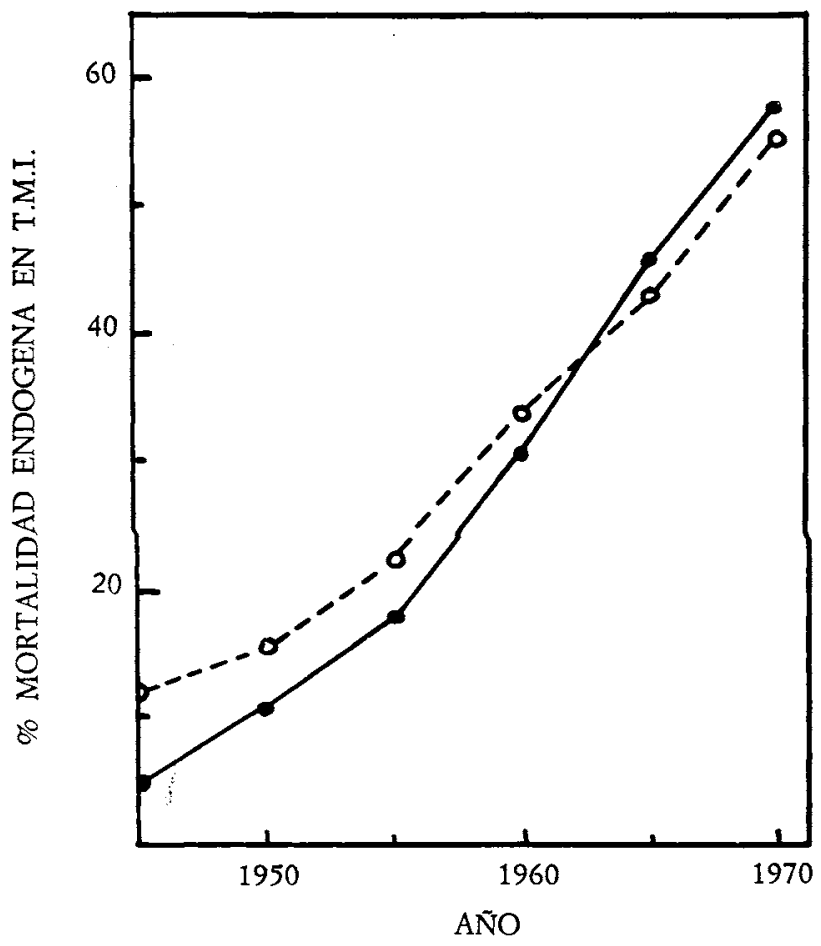

Madrid capital

- - - - E - España

Mortalidad diferencial según la edad del fallecimiento (periodos de vida)

Existe una clara relación entre los tipos de mortalidad según la etiología del fallecimiento y según la edad del fallecido, ya que las muertes por causa endógena se concentran durante el primer mes de vida (mortalidad neonatal), mientras que entre el mes y los once meses de vida (mortalidad postneonatal) la mortalidad se debe fundamentalmente a causas exógenas. Por ello, dado el importante cambio que desde el punto de vista de la etiología se ha producido durante el período estudiado, era previsible esperar repercusiones del mismo en la mortalidad por edad (cuadros 7 a 10).

En efecto, entre las tendencias de la mortalidad exógena y postneonatal se observa mucha similitud, del mismo modo que entre la endógena y neonatal 


\section{CUADRO 7}

Tasas de mortalidad infantil y submortalidades según la edad del fallecido. Proporciones de cada submortalidad en el total (Madrid capital)

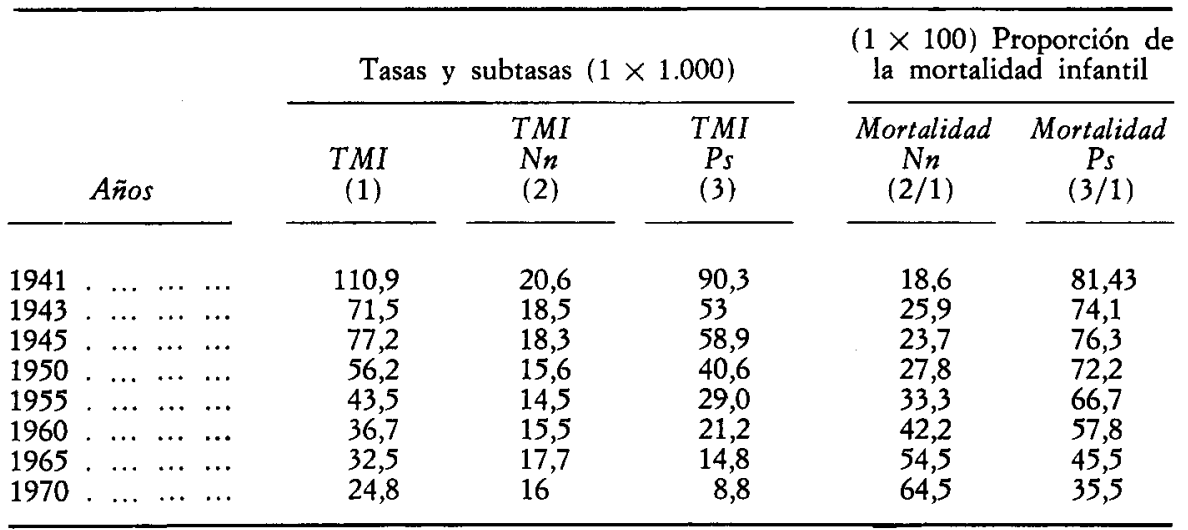

FuENTE: Elaboración propia en base a los MNP (INE).

Neonatal: $N n$.

Postneonatal: Ps.

\section{CUADRO 8}

Tasas de mortalidad infantil y submortalidades según la edad del fallecido. Proporciones de cada submortalidad en el total

(España)

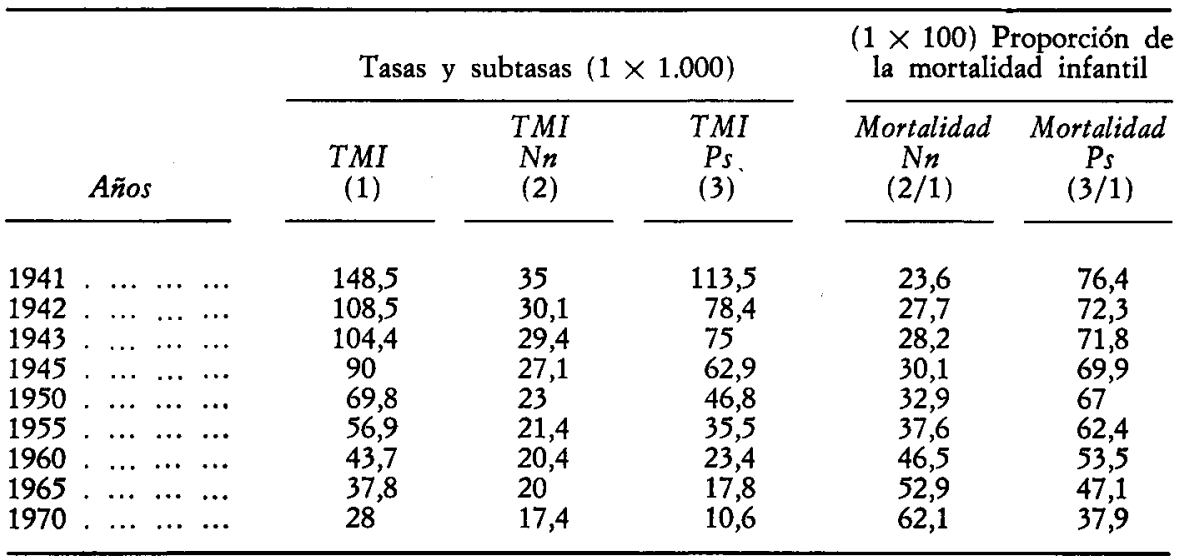

FUeNTE: Elaboración propia en base a los MNP (INE).

Neonatal: $N n$.

Postneonatal: Ps. 


\section{CUADRO 9}

Tasas de mortalidad infantil y submortalidades según la edad del fallecido. Proporciones de cada submortalidad en el total infantil

(Madrid resto provincia)

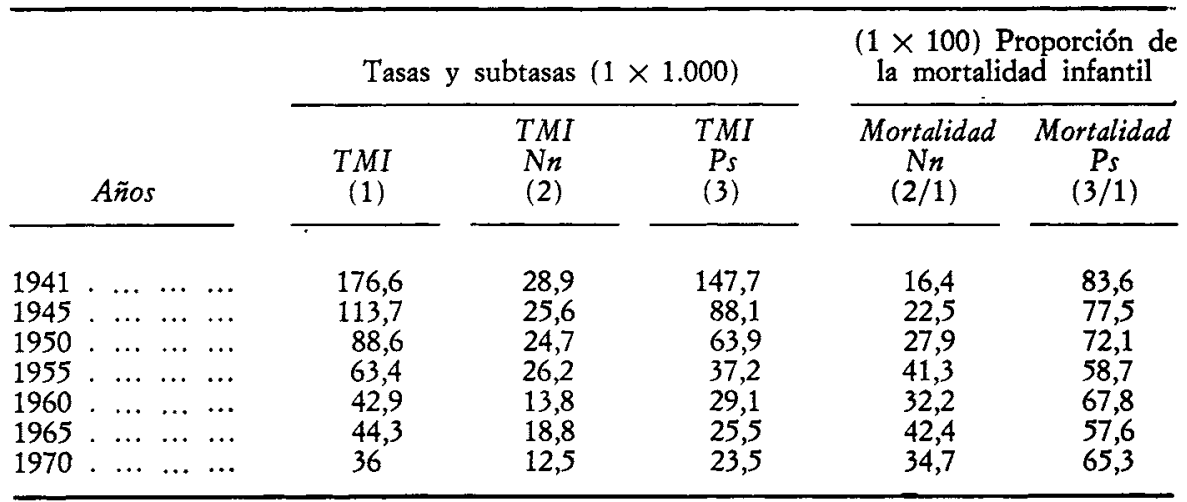

FUENTE: Elaboración propia en base a los $M N P$ (INE).

Neonatal: $N$.

Postneonatal: $P_{s}$.

\section{CUADRO 10}

Tasas de mortalidad infantil y submortalidades según la edad del fallecido. Proporciones de cada submortalidad en el total infantil

(Madrid provincia)

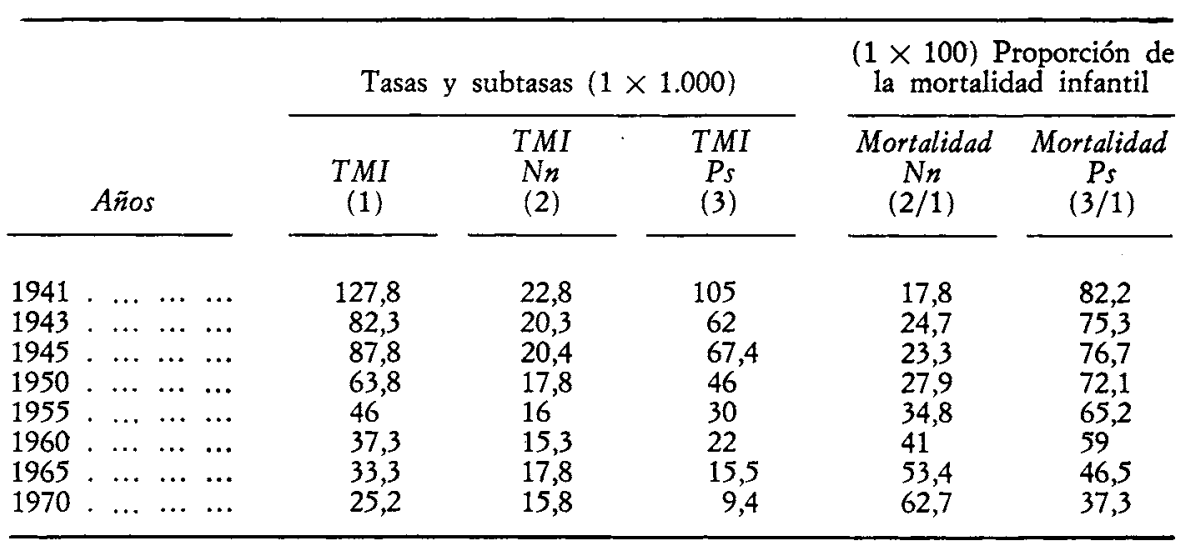

FUENTE: Elaboración propia en base a los MNP (INE).

Neonatal: $N n$.

Postneonatal: Ps. 
existen claros puntos en común. Sin embargo, hay notables diferencias en esos dos pares de submortalidades que se hace preciso analizar. Así, considerando el período globalmente, la tendencia en las submortalidades por edad es de descenso rápido en la mortalidad postneonatal, lo que coincide con la evolución de la mortalidad exógena (cuadros 3 a 6), como se ha observado. Descenso también, aunque muy gradual, en la neonatal (incluso estabilización en Madrid capital a partir de principios de los años cincuenta). La tendencia de esta última submortalidad es contraria a la de la mortalidad endógena, que durante el período analizado aumenta, lo que podría hacer dudar de la relación antes enunciada.

La explicación a esa aparente discordancia entre las tendencias de la mortalidad neonatal y la de origen endógeno se encuentra en que en la mortalidad neonatal, además de mortalidad por causa endógena, también existe por causa exógena, y el hecho de que el descenso de ésta sea muy rápido compensa el aumento de las muertes por aquella otra etiología.

Por lo expuesto, los cambios en el nivel de mortalidad diferencial entre el primer mes y el resto del año de vida, así como en sus tendencias —que se analizan seguidamente-, no son cambios de diferente naturaleza a los encontrados en la mortalidad endógena y exógena, sino consecuencias de la evolución de estas últimas. Por ello, carece de sentido analizar la mortalidad infantil según la edad sin antes relacionarla con las conclusiones halladas en el estudio de la mortalidad infantil desde el criterio de la etiología. Asimismo, las explicaciones argumentadas a este último respecto no serán desarrolladas de nuevo, sino que se harán extensibles a los cambios observados en la mortalidad por edad.

Por otra parte, del análisis de la magnitud del descenso de la mortalidad por edad se desprende que Madrid capital disminuye su mortalidad neonatal en un 18,1 por 100 de su valor, mientras la postneonatal lo hace en un 88,4 por 100 , situación ésta de desventaja en el descenso frente al total nacional, ya que si la mortalidad postneonatal se reduce en términos parecidos en España, su mortalidad neonatal logra alcanzar casi la mitad de su valor al inicio del período (cuadro 11 y gráfico 7$)^{19}$.

Esta diferencia negativa para la capital aparece en torno a 1960, fecha en la que se producen las interferencias estadísticas a las que ya se ha hecho referencia. No obstante, a pesar de este diferente titmo de descenso en los últimos dos ámbitos, el nivel de estas submortalidades en Madrid es menor que en España a lo largo de todo el período.

En el resto de la provincia de Madrid se presenta la misma tendencia al descenso de sus tasas, tanto en las muy altas postneonatales (muy por encima

${ }^{19}$ En los Movimientos Naturales de la Población existen datos de defunciones de menores de un año de edad por períodos de vida de 1900 a 1902, para luego interrumpirse esta información hasta 1941. Desde esta fecha aparecen regularmente hasta la actualidad. Analiza esos tres primeros años del siglo, para el total de España, M. PAscuA, La mortalidad infantil en España, Madrid, Dirección General de Sanidad, 1934, pp. 15-18. 


\section{CUADRO 11}

Tasas de mortalidad infantil según la edad del fallecido Tasas corregidas $(1 \times 1.000$ nacidos vivos $)$

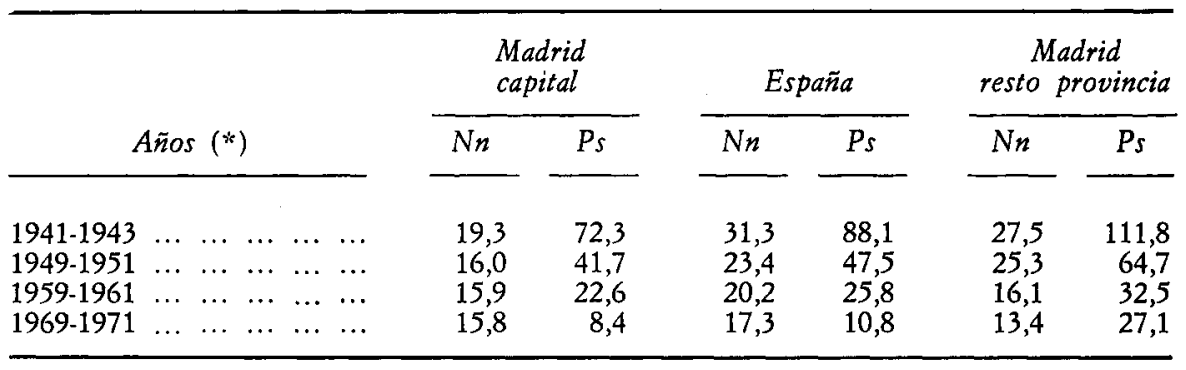

(*) Se utilizan las tasas medias de los trienios enumerados.

$N n=$ mortalidad neonatal.

Ps $=$ mortalidad postneonatal.

FuENTE: Elaboración propia en base a los Movimientos Naturales de la Población.

del total nacional y que justifican su altísima mortalidad infantil) como en las tasas neonatales, bajas para un medio predominantemente rural (durante buena parte del período) y por debajo de las de España. El ritmo del descenso en la mortalidad del primer mes de vida es el mismo que en el total nacional (50 por 100), mientras que en el resto del año de edad es más rápido ( $3 / 4$ partes de su valor).

La situación extrema que se presenta entre Madrid capital y el resto de la provincia es explicable por la transferencia de parte de la mortalidad neonatal de la provincia a la capital, donde tiene lugar, como consecuencia, una desaceleración -e incluso estabilización - del descenso de las tasas neonatales a partir de 1950 , mientras que la mortalidad postneonatal continúa cayendo.

Como consecuencia de la desigualdad en el ritmo de descenso de las dos submortalidades por edad, las altas tasas de mortalidad postneonatal al principio de los años cuarenta, que entonces superaban ampliamente a las neonatales, pasan a la posición contraria antes de finalizar el período, cuando la mortalidad neonatal es mayor que la postneonatal (cuadro 11 y gráfico 7 ).

Este proceso produce el tercer cambio importante en la evolución de la mortalidad infantil durante 1900-1970, que, como ya se ha señalado, no es más que la consecuencia del segundo cambio al que se hizo mención con anterioridad: por primera vez, en 1963, en Madrid capital (cuadro 12 y gráfico 8), y un año más tarde en España, se iguala la mortalidad neonatal a la postneonatal (en el resto de la provincia de Madrid no se había producido al 
finalizar el período). A partir de esas fechas, la proporción mayoritaria de muertes se produce durante el primer mes de vida. El predominio de mortalidad neonatal o postneonatal en una sociedad no es un dato baladí, pues corresponde a estadios demográficos y sociales diferentes.

\section{GRAFICO 7}

Evolución de la mortalidad infantil según la edad (períodos de vida) del fallecido, 1945-1970

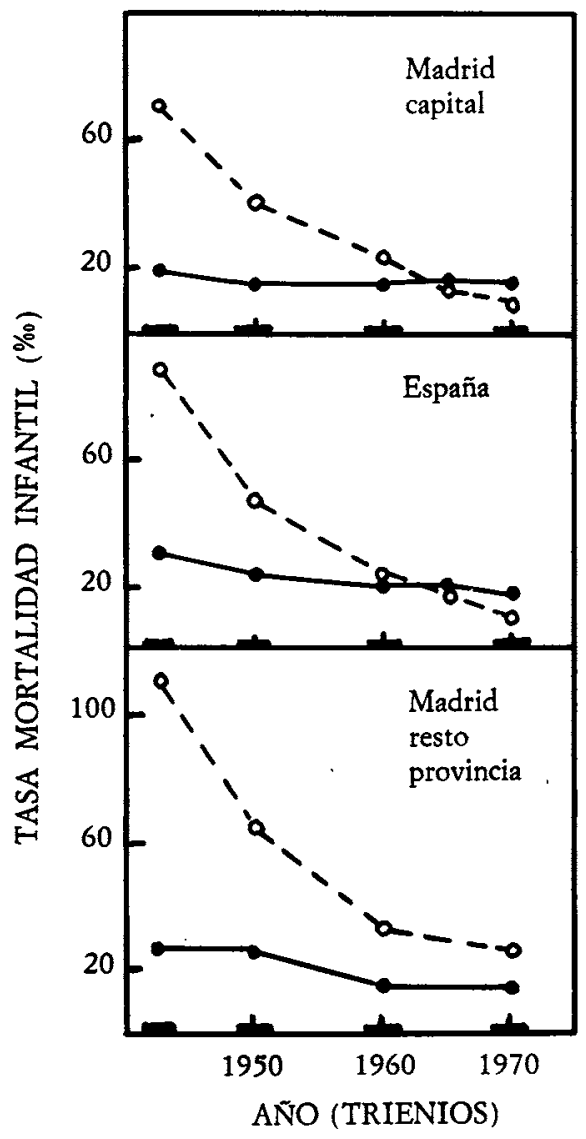

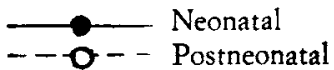




\section{CUADRO 12}

Tasas de mortalidad infantil según la edad del fallecimiento en Madrid capital Tasas corregidas $(1 \times 1.000$ nacidos vivos $)$

\begin{tabular}{|c|c|c|c|c|c|}
\hline$A \tilde{n} o s$ & Neonatal & Postneonatal & $A \tilde{n} o s$ & Neonatal & Postneonatal \\
\hline 1941 & 20,7 & 90,3 & 1957 & 12,3 & 30,5 \\
\hline 1942 & $-18,8$ & 73,6 & 1958 & 12,3 & 29,0 \\
\hline 1943 & 18,5 & 53,1 & 1959 & 13,5 & 24,4 \\
\hline 1944 & 17,6 & 58,6 & 1960 & 15,5 & 21,2 \\
\hline 1945 & 18,3 & 58,9 & 1961 & 18,7 & 22,3 \\
\hline 1946 & 17,4 & 57,1 & 1962 & 16,5 & 17,4 \\
\hline 1947 & 17,5 & 51,3 & 1963 & 16,7 & 17,3 \\
\hline 1948 & 14,7 & 41,8 & 1964 & 16,1 & 14,5 \\
\hline 1949 & 16,1 & 43,6 & 1965 & 17,7 & 14,8 \\
\hline 1950 & 15,8 & 40,6 & 1966 & 16,8 & 15,2 \\
\hline 1951 & 16,0 & 41,0 & 1967 & 17,4 & 10,9 \\
\hline 1952 & 18,1 & 40,6 & 1968 & 17,7 & 10,6 \\
\hline 1953 & 16,2 & 36,3 & 1969 & 17.1 & 9,5 \\
\hline 1954 & 16,0 & 36,0 & 1970 & 16,0 & 8,8 \\
\hline 1955 & 14,5 & 29,0 & 1971 & 14,3 & 6,8 \\
\hline 1956 & 14,6 & 32,1 & & & \\
\hline
\end{tabular}

Fuente: Elaboración propia en base a los Movimientos Nacionales de la Población de España.

Como consecuencia de este cambio, aparece con nitidez una relación inversa entre la edad del fallecido y el nivel de mortalidad infantil de su grupo de edad.

\section{Mortalidad infantil durante el primer día de vida}

La relación inversa observada en la mortalidad de los menores de un año entre la edad del fallecido y el nivel de mortalidad del grupo de edad al que pertenecía hizo suponer que la mayor dificultad en la reducción de la mortalidad infantil podría encontrarse en las primeras horas de vida.

Para verificar dicha hipótesis se ha recurrido a analizar la evolución de la mortalidad que se produce en el curso del parto (intranatal) y durante el primer día de vida (postnatal) ${ }^{20}$, desde que se dispone de los datos pertinentes

${ }^{20}$ La tasa intranatal o postnatal es el número de defunciones que se producen «al nacer" o durante las "primeras 24 horas de vida», respectivamente, por cada 1.000 nacidos vivos. Por lo tanto, dichas tasas no equivaleỉ a las que con el mismo nombre utiliza, entre otros autores, A. ARBELo, La mortalidad, op. cit., pp. 31-35, ya que este autor relaciona los sucesos con el total de nacidos (vivos y muertos). Se ha creído oportuno utilizar los indicadores definidos porque en este estudio se analiza la mortalidad infantil, fenómeno que se estudia a través del suceso del fallecimiento, cuya premisa básica para que este último se produzca es otro suceso: el nacimiento vivo. Asimismo, esto hace que 


\section{GRAFICO 8}

Evolución de la mortalidad infantil según la edad (periodos de vida) del fallecido en Madrid capital, 1941-1970

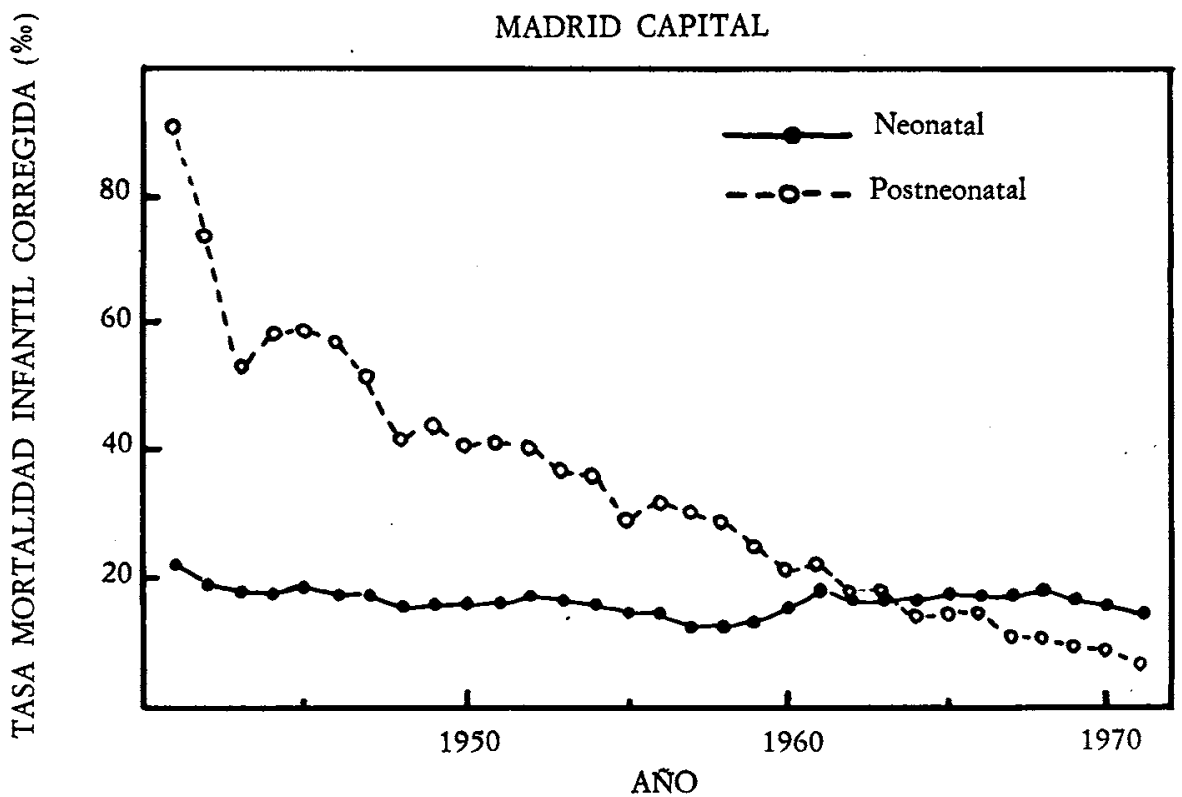

en los Movimientos Naturales de la Población para el cálculo de sus tasas (1930).

Asimismo, de haber sido posible, se habría analizado la mortalidad que acontece durante la primera semana de vida (precoz), pero ello no es posible para España por no disponer de datos sobre las .defunciones que se producen en este período de vida, sin duda uno de los más interesantes dentro del fenómeno que se estudia.

Del análisis de aquellas dos submortalidades se deduce, en general, una tendencia a la estabilidad en los tres ámbitos. Sin embargo, es evidente que las tasas intranatales $\mathrm{y}$, sobre todo, postnatales aumentan rápidamente en 1960 , lo que, sin duda, se debe a la corrección en dicha fecha de buena parte del subregistro existente con anterioridad, y al que se ha hecho referencia repetidamente.

dichas submortalidades sean comparables con el resto de las que componen la mortalidad infantil, permitiendo evaluar su peso dentro del total de la mortalidad de los menores de un año. El mismo autor citado, A. ARBELo, en su posterior obra Demografía sanitaria, op. cit., pp. 114-116, siguiendo las indicaciones de la Organización Mundial de la Salud (OMS), ya utiliza los indicadores descritos, es decir, aquellos en cuyo denominador sólo se consideran los nacidos vivos. 
El hecho de que la notable disminución de dicho error se haya observado en la mortalidad endógena, y ahora en la intranatal y muy especialmente en la postnatal, parece indicar que tal subregistro se debía fundamentalmente a la mortalidad producida en el primer día de vida. Este error registral induce a pensar que pudo haber existido una tendencia a certificar parte de las defunciones intranatales y postnatales como producidas con posterioridad ${ }^{21}$. A esta causa, dependiente del criterio del profesional, y que disminuye ante la exigencia de una mayor exactitud en la declaración del momento del fallecimiento (en 1960), se debería la brusquedad del cambio. Por desgracia, dicha explicación no se puede respaldar cuantitativamente por no disponerse de datos desagregados de defunciones infantiles por períodos dentro del primer mes de vida.

Por lo tanto, a través del análisis de las series de mortalidad intranatal y postnatal ha sido posible observar rupturas en algunos momentos de su evolución, que ayudan a interpretar alteraciones observadas anteriormente en la tendencia de la mortalidad infantil según otros criterios de análisis.

Con independencia de la interferencia puramente estadística descrita, del análisis de la mortalidad próxima al nacimiento se deducen algunas conclusiones destacables. La tendencia a la estabilidad de la mortalidad durante el parto presenta diferencias de nivel entre Madrid y España —más alto en esta última- durante el período, que se hacen despreciables al final del mismo (cuadro 13 y gráfico 9). La situación privilegiada de la capital en cuanto a contar con servicios especializados de medicina, como ya se ha dicho, se refleja en sus tasas más bajas, así como en las del resto de la provincia, que indirectamente se beneficia de esos servicios al desplazar los partos previsiblemente difíciles a la capital.

Dentro de esta tendencia a la estabilidad existen períodos durante los cuales disminuye la mortalidad intranatal; por ejemplo, en la década de los años cincuenta en Madrid capital esta mortalidad desciende notablemente. No obstante, en el futuro la reducción del nivel de mortalidad intranatal es probable que siga un proceso lento. Ténganse en cuenta los cambios que se han producido en el medio social madrileño desde 1930 a 1960 y las escasas diferencias conseguidas y mantenidas en esta submortalidad (cuadro 14).

Por último se analiza la mortalidad postnatal, en la que también se encuentra cierta tendencia a la estabilidad (cuadro 13 y gráfico 9). Al principio del período estudiado, las tasas en la capital son más bajas que en el resto de la provincia y que en España. Más tarde sobreviene el cambio de tendencia

"No obstante, no se puede descartar que una pequeña proporción de este subregistro se desplazase a engrosar el apartado de «nacidos muertos». Pero si bien es cierto que las defunciones anteriores al alumbramiento («nacidos muertos») disminuyen en relación a los «nacidos vivos» en esas fechas, ello no supone un punto de ruptura, sino, al contrario, la continuación de la tendencia al descenso que caracteriza a la mortalidad prenatal durante el período. Esta «mortalidad» anterior al nacimiento también fue estudiada para el mismo período en la Memoria de Licenciatura de la autora, ya citada, cap. V, 4, pp. 177-181. 


\section{CUADRO 13}

Mortalidades intranatal y postnatal

Tasas corregidas $(1 \times 1.000$ nacidos vivos $)$

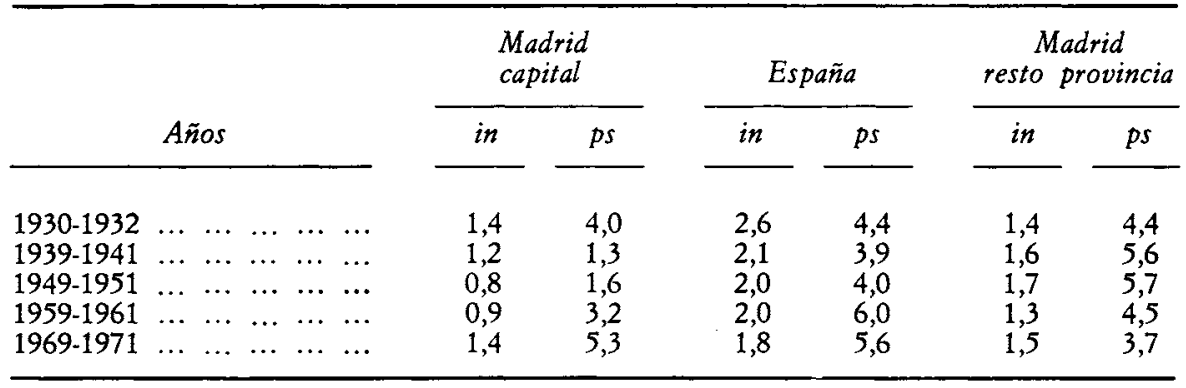

Fuente: Elaboración propia en base a los $M N P$.

in = mortalidad intranatal.

$p s=$ mortalidad postnatal (primer día de vida).

\section{GRAFICO 9}

Evolución de la mortalidad intranatal y postnatal, 1930-1970

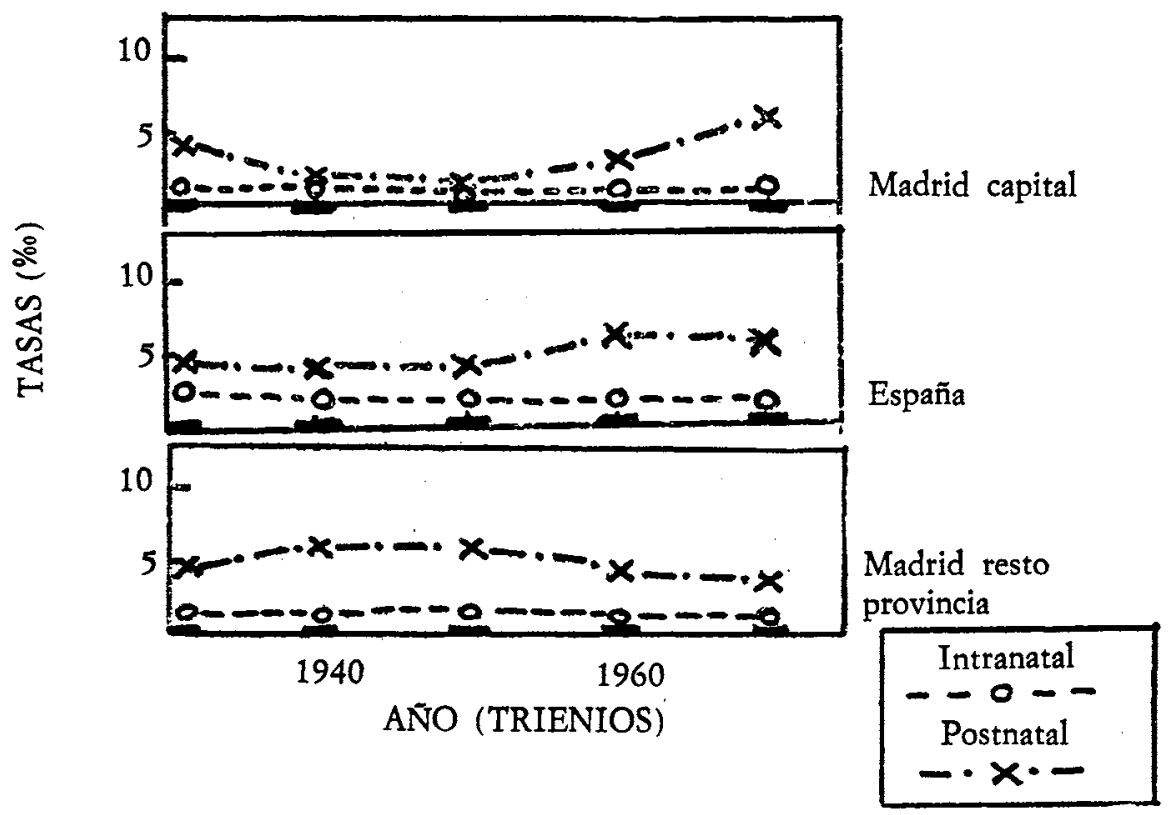




\section{CUADRO 14}

Mortalidades intranatal y postnatal en Madrid capital Tasas corregidas $(1 \times 1.000$ nacidos vivos $)$

\begin{tabular}{|c|c|c|c|c|c|}
\hline$A \tilde{n} o s$ & Intranatal & Postnatal & Años & Intranatal & Postnatal \\
\hline 1930 & 1,28 & 6,51 & 1951 & 0,82 & 1,93 \\
\hline 1931 & 1,65 & 3,18 & 1952 & 1,26 & 1,77 \\
\hline 1932 & 1,38 & 2,42 & 1953 & 0,55 & 1,58 \\
\hline 1933 & 1,21 & 2,90 & 1954 & 0,87 & 1,31 \\
\hline 1934 & 0,75 & 1,36 & 1955 & 0,90 & 1,67 \\
\hline 1935 & 1,64 & 0,59 & 1956 & 0,44 & 0,88 \\
\hline 1936 & 0,50 & 1,32 & 1957 & 0,30 & 0,58 \\
\hline 1937 & 5,33 & 1,12 & 1958 & 0,22 & 0,39 \\
\hline 1938 & 2,65 & 1,54 & 1959 & 0,36 & 0,26 \\
\hline 1939 & 0,47 & 1,17 & 1960 & 1,10 & 3,72 \\
\hline 1940 & 2,13 & 0,60 & 1961 & 1,29 & 5,78 \\
\hline 1941 & 0,99 & 2,13 & 1962 & 1,64 & 4,97 \\
\hline 1942 & 1,18 & 1,43 & 1963 & 1,29 & 5,00 \\
\hline 1943 & 0,84 & 2,28 & 1964 & 1,05 & 4,13 \\
\hline 1944 & 0,86 & 2,17 & 1965 & 1,24 & 4,74 \\
\hline 1945 & 1,02 & 1,68 & 1966 & 1,04 & 4,67 \\
\hline 1946 & 0,43 & 1,28 & 1967 & 1,17 & 5,98 \\
\hline 1947 & 1,33 & 1,50 & 1968 & 1,23 & 6,03 \\
\hline 1948 & 1,93 & 1,46 & 1969 & 0,91 & 5,66 \\
\hline 1949 & 0,90 & 1,52 & 1970 & 2,11 & 5,23 \\
\hline 1950 & 0,62 & 1,52 & 1971 & 1,18 & 4,98 \\
\hline
\end{tabular}

FUENTE: Elaboración propia en base a los MNP.

observado ya en la mortalidad neonatal, por el que el menor nivel de mortalidad se logra en el resto de la provincia, incluso por debajo de la capital, que casi se iguala con España en su conjunto. Este descenso de nivel en la mortalidad postnatal de la provincia respecto a la de la capital, y por tanto la existencia de una hipermortalidad infantil en esta última, se debe a que aquélla transfiere población de alto riesgo a la capital por las razones ya indicadas.

En el análisis desagregado que se acaba de realizar destaca, pues, la existencia de una tendencia a la estabilidad en la mortalidad durante el nacimiento y primer día de vida. Por consiguiente, parece confirmarse que cuanto más cerca se está del momento del nacimiento, más dificultades ofrece la disminución de la mortalidad infantil y mayor es su impermeabilidad a los cambios socioeconómicos y sanitarios que tienen lugar en el medio observado. 
Relación entre la mortalidad diferencial según la etiología del fallecimiento y la mortalidad diferencial según la edad del fallecido

A través del análisis realizado es evidente la existencia de una relación entre la etiología del fallecimiento y la edad del fallecido, por lo que se creyó oportuno medir lo más ajustadamente posible esa relación, ya que sus indicadores, como se ha visto, no son sustituibles ni sus niveles y tendencias son iguales.

Ciertamente, basándose en estudios realizados sobre países con buenas estadísticas, se parte de la hipótesis de que a partir del primer mes de vida la mortalidad endógena es despreciable en el primer año de edad y que, sin embargo, la mortalidad exógena se reparte, aunque muy desigualmente, entre la mortalidad neonatal (un 20 por 100) y postneonatal (el 80 por 100 restante de la mortalidad por causa exógena). Además, la tesis sostenida por algunos autores afirma que esta distribución es estable ${ }^{22}$, llegando a mantener casi una total independencia del nivel de las condiciones sanitarias alcanzadas. No obstante, no se creyó pertinente aceptar esta generalización de la relación que ahora se analiza sin verificarla previamente.

En primer lugar, se observa que a principios del período el peso mayoritario en la mortalidad neonatal lo ocupaba la mortalidad por causa exógena, pero debido a la rápida caída de este tipo de muertes queda sustituida su preponderancia por la endógena (cuadro 15 y gráfico 10 ), proceso que se encontró igualmente en la mortalidad infantil en su conjunto. El resultado de este cambio es que si la mortalidad exógena en 1945 representaba en la neonatal de Madrid el 79 por 100 (en España, 60 por 100), en 1970 la mortalidad endógena la supera alcanzando el 89,3 por 100 (en España, 88,3 por 100). Esta sustitución explica la diferencia hallada en el anterior apartado entre las tendencias de la mortalidad endógena (en aumento) y neonatal (en descenso), ya que esta diferencia no se debe sólo a que la fuerte caída de la mortalidad exógena compense el aumento de mortalidad por causa endógena dentro de la neonatal, sino también a la alta proporción que representa aquella etiología dentro de la mortalidad de los menores de un mes de vida, durante la mayor parte del período (cuadro 15).

Por otra parte, del análisis de la relación concreta de la mortalidad exógena con las dos submortalidades por edad (cuadro 16) es de destacar que, a pesar del fuerte descenso de la mortalidad de origen exógeno en el período, la proporción en que se distribuye este tipo de mortalidad entre los períodos de vida del fallecido varía con extremada lentitud, manteniéndose una relativa estabilidad en torno al 20 por 100 en la neonatal y al 80 por 100 en la postneonatal. No obstante, existe una tendencia débil y gradual consistente en que

${ }^{22} \mathrm{~J}$. Bourgeors-Pichat, «La mesure de la mortalité infantile. I. Principes et méthodes", Population, núm. 4, abril-junio 1951, pp. 239 y 241. Tesis aplicada por R. Pressat, El análisis, op. cit., p. 151, y M. Poulain y D. Tabutin, «La mortalité», op. cit., p. 129 (nota 8). 


\section{CUADRO 15}

Proporciones que representan las mortalidades endógena y exógena en la mortalidad neonatal total

$(1 \times 100)$ Mortalidad neonatal $=100$

\begin{tabular}{|c|c|c|c|c|c|c|c|c|}
\hline \multirow[b]{2}{*}{ Años } & \multicolumn{4}{|c|}{ MORTALIDAD ENDOGENA } & \multicolumn{4}{|c|}{ MORTALIDAD EXOGENA } \\
\hline & España & $\begin{array}{c}\text { Madrid } \\
\text { cap. }\end{array}$ & $\begin{array}{c}\text { Madrid } \\
\text { prov. }\end{array}$ & $\begin{array}{c}\text { Madrid } \\
\text { resto } \\
\text { prov. }\end{array}$ & España & $\begin{array}{c}\text { Madrid } \\
\text { cap. }\end{array}$ & $\begin{array}{c}\text { Madrid } \\
\text { prov. }\end{array}$ & $\begin{array}{c}\text { Madrid } \\
\text { resto } \\
\text { prov. }\end{array}$ \\
\hline $\begin{array}{llll}1941 & \cdots & \ldots \\
1943 & . & \ldots & \ldots \\
1945 & . & \ldots & \ldots \\
1950 & \cdot & \ldots & \ldots \\
1955 & \cdots & \ldots & \ldots \\
1960 & \cdots & \ldots & \ldots \\
1965 & . & \ldots & \ldots \\
1970 & \ldots & \ldots\end{array}$ & $\begin{array}{l}33,6 \\
40 \\
47 \\
59 \\
72,5 \\
80,9 \\
88,3\end{array}$ & $\begin{array}{l}28 \\
21 \\
37,4 \\
52 \\
72 \\
83,9 \\
89,3\end{array}$ & $\begin{array}{l}22 \\
35,3 \\
54,2 \\
69,7 \\
83 \\
88,3\end{array}$ & $\begin{array}{l}62 \\
52 \\
73,4 \\
55\end{array}$ & $\begin{array}{l}66,4 \\
60 \\
53 \\
41 \\
27,5 \\
19,1 \\
11,7\end{array}$ & $\begin{array}{l}72 \\
79 \\
62,6 \\
48 \\
28 \\
16,1 \\
10,7\end{array}$ & $\begin{array}{l}78 \\
64,7 \\
45,8 \\
30,3 \\
17 \\
11,7\end{array}$ & $\begin{array}{l}38 \\
48 \\
26,6 \\
45\end{array}$ \\
\hline
\end{tabular}

FUENTE: Elaboración propia en base a los MNP.

\section{GRAFICO 10}

Evolución de la proporción representada por la mortalidad endógena en la mortalidad neonatal, 1945-1970

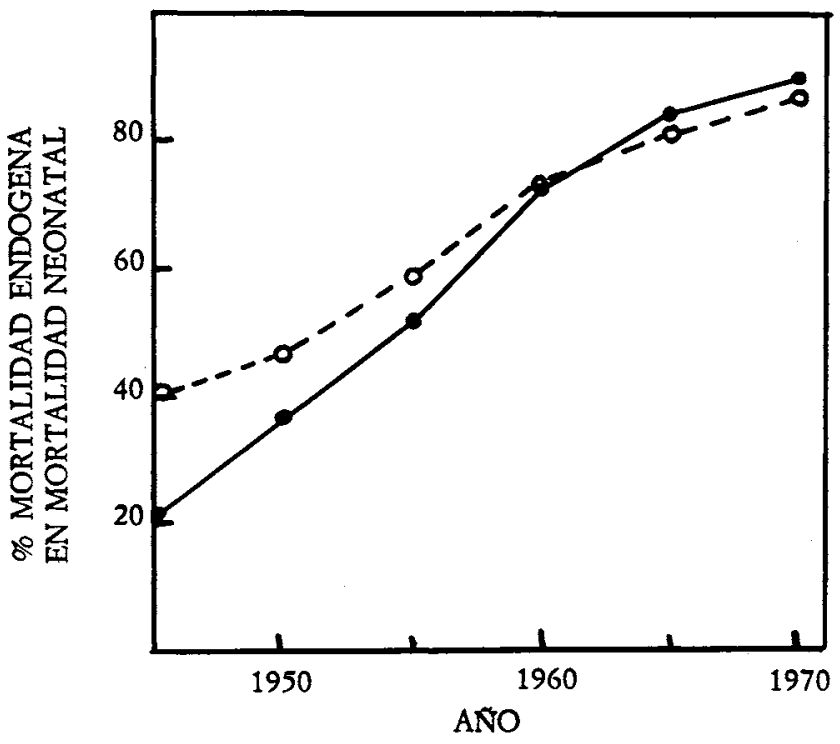

$\underset{-0--}{\longrightarrow}$ Madrid capital 


\section{CUADRO 16}

Proporciones que representan las mortalidades neonatal y postneonatal en la mortalidad exógena total

$(1 \times 100)$ Mortalidad exógena $=100$

\begin{tabular}{|c|c|c|c|c|c|c|c|c|}
\hline \multirow[b]{2}{*}{$A \tilde{n} o s$} & \multicolumn{4}{|c|}{ MORTALIDAD NEONATAL } & \multicolumn{4}{|c|}{ MORTALIDAD POSTNEONATAL } \\
\hline & España & $\begin{array}{c}\text { Madrid } \\
\text { cap. }\end{array}$ & $\begin{array}{c}\text { Madrid } \\
\text { prov. }\end{array}$ & $\begin{array}{c}\text { Madrid } \\
\text { resto } \\
\text { prov. }\end{array}$ & España & $\begin{array}{c}\text { Madrid } \\
\text { cap. }\end{array}$ & $\begin{array}{c}\text { Madrid } \\
\text { prov. }\end{array}$ & $\begin{array}{c}\text { Madrid } \\
\text { resto } \\
\text { prov. }\end{array}$ \\
\hline$\ldots \ldots$ & & & & & & & & \\
\hline $\begin{array}{ll}\ldots & \ldots \\
\ldots & \cdots\end{array}$ & 20,7 & 20 & 20,4 & & 79,3 & 80 & 79,6 & \\
\hline$\ldots$ & 20,4 & 19,8 & & & 79,6 & 80,2 & & \\
\hline$\ldots \ldots$ & 20,6 & 19,6 & 20 & & 79,4 & 80,4 & 80 & \\
\hline $\begin{array}{ll}\ldots & \ldots \\
\ldots & \ldots\end{array}$ & 19,8 & 19,3 & 19,6 & 21,2 & 80,2 & 80,7 & 80,4 & 78,8 \\
\hline$\ldots \ldots$ & 19,2 & 17,3 & 17,4 & 18,5 & 80,8 & 82,7 & 82,6 & 81,5 \\
\hline$\ldots$ & 17,7 & 16,4 & 16,3 & 16,3 & 82,3 & 83,6 & 83,7 & 83,7 \\
\hline $1970 \ldots \ldots$ & 16,2 & 16,2 & 16,5 & 19,4 & 83,8 & 83,8 & 83,5 & 80,6 \\
\hline
\end{tabular}

FUENTE: Elaboración propia en base a los MNP.

la proporción de mortalidad por causa exógena que se produce en el período postneonatal sea cada vez mayor (gráfico 11) y, por tanto, que en la mortalidad neonatal disminuya a lo largo de los años estudiados.

Se trata, pues, de una lenta transferencia de parte del porcentaje representado por la mortalidad exógena dentro de la mortalidad neonatal al representado en la postneonatal.

Por lo expuesto, no es posible aceptar como invariable la cuantificación citada sobre la distribución de la mortalidad exógena en los dos períodos de vida analizados, si no se fija un umbral mínimo a partir del cual se cumpla ni se determina espacial y temporalmente dicha afirmación.

En el caso de Madrid capital, y también de España, la hipotética invariabilidad de esa distribución de las defunciones de etiología exógena sólo sería aceptable hasta 1955, para posteriormente, coincidiendo con el inicio de un cambio socioeconómico y de mejores condiciones higiénico-sanitarias, disminuir la proporción de mortalidad exógena en la neonatal y, obviamente, aumentar su peso en la postneonatal. Así, en 1970, tanto en Madrid capital como en España, la mortalidad neonatal participaba en algo más del 16 por 100 del total de la mortalidad infantil exógena y, como consecuencia, la mortalidad postneonatal absorbía casi el 84 por 100 . Esto contrasta con la situación de 1945, que se adaptaba a la tesis referida. En cuanto al resto de la provincia de Madrid, se mantienen durante todo el período valores aproximados a la norma citada, pero con frecuentes irregularidades ya habituales en este ámbito. 


\section{GRAFICO 11}

Evolución de la proporción representada por la mortalidad postneonatal en el total de la mortalidad exógena, 1945-1970

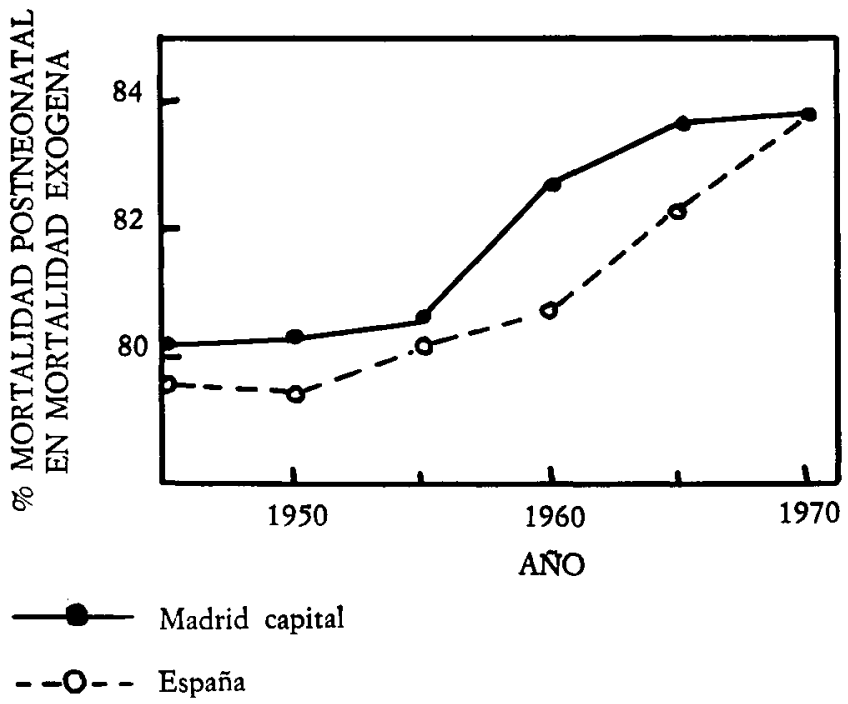

Se concluye de lo dicho que se tiende a una identificación cada vez mayor entre los dos pares de submortalidades por edad y etiología a los que se ha venido haciendo referencia (el porcentaje de mortalidad endógena en la neonatal aumenta, lo que supone una creciente concentración de la mortalidad exógena en la postneonatal). Esta tendencia a la identificación se encuentra en Madrid capital en una situación más avanzada que en España en su conjunto, salvo en el último año del período, en que se igualan ambos medios, mientras que en el resto de la provincia se observan las mismas tendencias con proporciones medias entre la capital y el total nacional.

Asimismo, de lo expuesto se deduce que no sólo resultan desigualmente afectados por la mortalidad infantil los dos períodos de vida tratados, sino que las perspectivas del descenso futuro de sus respectivas mortalidades - a partir del período estudiado- son aún más desiguales. Ello se debe, en último término, al creciente peso que las muertes de tipo endógeno han ido adquiriendo en la mortalidad infantil. 


\section{CONCLUSION}

La evolución de la mortalidad infantil de la provincia de Madrid durante el período 1900-1970, en general, corre paralela a la tendencia seguida por España en su conjunto, presentando un acusado descenso. En efecto, la magnitud de este descenso y el ritmo acelerado con que tiene lugar permiten reducir la mortalidad infantil en cerca del 90 por 100 de su valor inicial en sólo setenta años, a pesar de las interrupciones del proceso por crisis demográficas, entre las que destacan la pandemia de gripe de 1918 y la guerra civil de 1936-1939.

Un estudio pormenorizado del fenómeno permite comprobar que esta caída encierra cambios de tendencias en los componentes del fenómeno estudiado, así como en las relaciones existentes entre ellos. Ciertamente, la caída de la mortalidad infantil se debe a un rápido descenso de la mortalidad causada por enfermedades de origen exógeno, mientras que la mortalidad por causa endógena, durante el período en que es posible su análisis en España (1945-1970), coincide con una de las oscilaciones - que abarca la etapa de ascenso y estabilización de sus tasas - a las que no es ajena la evolución de este tipo de mortalidad. Este desigual ritmo de descenso de la mortalidad infantil según la etiología del fallecimiento tiene como consecuencia el que la mortalidad por causa endógena, que había tenido siempre un peso minoritario en el total de las muertes infantiles, llegue a ser la causa dominante al final del período.

Asimismo, una vez superadas las malas condiciones de vida de las capitales y debido al descenso de la mortalidad exógena, la mortalidad infantil diferencial desde un criterio ecológico sufre un cambio en su tendencia a mediados de los años veinte. A partir de esos años, la hipermortalidad infantil -hasta entonces característica de las capitales - se produce en el resto de las provincias.

Ese importante cambio en la mortalidad diferencial por etiología del fallecimiento constituye la base de cambios observados en la mortalidad de los menores de un año según la edad del fallecido ${ }^{23}$.

En efecto, cuando el estudio de la mortalidad infantil se aborda desde el criterio de la edad, se encuentra una mortalidad diferencial basada en una relación inversa entre la edad del fallecido y el nivel de mortalidad del grupo de edad al que pertenecía. Dividiendo la mortalidad infantil en sus dos más importantes grupos de edad, neonatal y postneonatal, este último había tenido siempre un nivel más alto de mortalidad hasta que en la década de los sesenta es sustituido por el neonatal; desde entonces, este grupo de edad mantiene el peso mayoritario en la mortalidad infantil, cambio igual al que tuvo lugar en la mortalidad por etiología del fallecimiento.

${ }^{23}$ También tiene consecuencias en la mortalidad infantil según el sexo del fallecido, tema tratado ampliamente en la Memoria de Licenciatura de la autora, cap. VII, pp. 223-228. 
Tal coincidencia tiene su explicación en el hecho de que la mortalidad infantil por causa endógena se concentra en el primer mes de vida, mientras que la mortalidad postneonatal absorbe la mayor parte de las muertes debidas a causas exógenas. Además, esta asociación entre estos dos pares de submortalidades por etiología y edad parecen evolucionar hacia una lenta pero creciente identificación. Asimismo, este proceso de identificación pone de relieve la inexactitud de una supuesta estabilidad en la distribución de la mortalidad exógena entre los dos períodos de vida citados.

Como consecuencia de la relación existente entre la edad del fallecido y la etiología del fallecimiento, los éxitos conseguidos frente a la mortalidad infantil son mayores cuanto más lejos del nacimiento ocurre el deceso, siendo la mortalidad durante el parto y el primer día de vida la que, por su naturaleza fundamentalmente endógena, ofrece mayor resistencia al cambio, y de ahí su tendencia a la estabilidad durante el período en estudio, poniendo en evidencia su relativa impermeabilidad a transformaciones sociales.

De lo expuesto se puede concluir que la mortalidad infantil desciende extraordinariamente durante el período observado, pero no menos importante es el hecho de que el descenso sostenido de esta mortalidad específica encubre las diversas evoluciones de las submortalidades en que se desagrega el fenómeno. Ello ha podido observarse a través del análisis pormenorizado llevado a cabo, gracias al cual se puede comprobar que la mortalidad infantil de finales del período no sólo ha disminuido su nivel, sino que tiene una estructura muy distinta a la de principios de siglo. 